\title{
Turbulent channel flow with either transverse or longitudinal roughness elements on one wall
}

\author{
By P. ORLANDI ${ }^{1}$, S. LEONARDI AND R. A. ANTONIA \\ ${ }^{1}$ Dipartimento di Meccanica e Aeronautica Università La Sapienza, Via Eudossiana 18, \\ 00184, Roma, Italy \\ ${ }^{2}$ Discipline of Mechanical Engineering, University of Newcastle, NSW 2308 Australia
}

(Received 7 January 2005 and in revised form 18 January 2006)

Direct numerical simulation results are presented for turbulent channel flows with two-dimensional roughness elements of different shapes. The focus is mainly on a geometry where the separation between consecutive roughness elements is small and for which the rate of change of the roughness function with respect to the separation between consecutive elements is large. Roughness elements are placed either along the flow direction or orthogonally to it. In the latter case, the drag is increased. For the former case, the possibility of drag reduction reflects the different relative contributions from viscous and Reynolds shear stresses. The Reynolds shear stress depends on the shape of the surface more than the viscous stress and is closely related to the near-wall structures. For orthogonal elements, there is no satisfactory correlation between the roughness function and parameters describing the roughness geometry. On the other hand, a satisfactory collapse of the data is achieved when the roughness function is plotted against the root mean square wall-normal velocity averaged over the plane of the roughness crests. Relative to a smooth wall surface, the Reynolds stress tensor near the wall tends to become more isotropic when the elements are orthogonal to the flow and less isotropic when the elements are aligned with the flow. The interdependencies between the departure from isotropy in the wall region, the organization of the wall structures, and the magnitude of the drag are assessed by examining the rotational component of the turbulent kinetic energy production and the probability density function of the helicity density.

\section{Introduction}

The effect of roughness may be thought to cause, in a broad sense, either an increase or a reduction in drag. The former case is usually associated with either three-dimensional or transverse two-dimensional roughness elements, while the latter is generally achieved with the use of riblets (elements aligned to the flow). Nikuradse (1933) was the first to investigate the Reynolds-number dependence of the drag for flows over a uniform sand grain roughness. Clauser (1954) showed that the effect of the roughness was to shift the velocity distribution in the log-region according to

$$
U^{+}=\kappa^{-1} \ln \left(y^{+}\right)+B-\Delta U^{+},
$$

where $\kappa$ is the Kármán constant, $B$ is a constant (equal to about 5.5 for channel flows) and $\Delta U^{+}$is the so-called roughness function ( + denotes normalization by wall variables, i.e. the frictional velocity $U_{\tau}$ and the kinematic viscosity $v$ ) which 
can depend on various characteristics of the roughness. Because of the wide range of roughnesses encountered in nature, it is difficult to define geometrical parameters which can represent all types of surface adequately. For many rough surfaces, $\Delta U^{+}$ depends on $k^{+}$, where $k$ is the height of the elements. Indeed, for sand grain roughness, a good fit to the data is provided by $\Delta U^{+}=\ln \left(1+0.3 k_{s}^{+}\right)\left(k_{s}\right.$ is a typical length related to the sand grain size). For a two-dimensional roughness, a similar behaviour has been found, namely $\Delta U^{+}=\kappa^{-1} \ln k^{+}+C$, where $C$ depends on the type of surface and roughness density. For a particular rough surface, usually referred to as 'd-type' (Perry, Schofield \& Joubert 1969), $\Delta U^{+}$is not supposed to depend on $k^{+}$. Instead, the dependence is on $d^{+}$, where $d$ represents either the diameter of the pipe, the thickness of the boundary layer or the half-width of a duct. To our knowledge, this dependence has yet to be confirmed. Using direct numerical simulations (DNSs), Leonardi et al. (2003a) showed that for transverse two-dimensional square elements, the maximum value of $\Delta U^{+}$occurred for $w / k=7$, where $w$ is the streamwise width of the cavity between consecutive elements. The DNS allows both the viscous drag and form drag to be estimated reliably. By averaging over one wavelength, the form drag is maximum whilst the viscous drag is negligible for $w / k=7$. The experimental results of Furuya, Miyata \& Fujita (1976) for circular rods also indicated that $\Delta U^{+}$ is maximum for $w / k=7$. For small values of $w / k$ (Leonardi et al. 2003b), there are large differences in $\Delta U^{+}$between rods and square bars, implying that it would be difficult, if not impossible, to find a dependence of $\Delta U^{+}$on geometrical parameters which would account for all rough surfaces. Orlandi et al. (2003) showed that using a non-zero wall-normal velocity as a boundary condition allowed the main effects of the roughness on the outer flow to be reproduced satisfactorily. It was consequently speculated that $\left\langle u_{2}^{\prime 2}\right\rangle$ could provide a better way of parameterizing the roughness (a prime indicates a velocity fluctuation, angule brackets denote averaging with respect to time as well as streamwise and spanwise directions). This speculation is tested in the present paper by carrying out DNSs of turbulent channel flows with square, circular and triangular two-dimensional elements placed on the bottom wall along the spanwise direction.

A different kind of roughness, aligned to the flow direction, may produce a drag reduction. Beckert \& Bartenwerfer (1989) reproduced shark skins in the laboratory and demonstrated that drag reduction could be achieved. Choi, Moin \& Kim (1993) performed a DNS of the flow above triangular riblets. They pointed out that drag reduction is obtained when the streamwise vortices cannot penetrate the space between the riblets. Since the turbulence-producing eddies have a diameter of approximately 20 wall units, they can reach the lateral walls if the lateral separation between riblets is larger than this distance and a drag increase results. Although the eddies produce a local increase in skin friction near the tip of riblets with dimension $S^{+}=15$ ( $S$ is the height of the riblets), the skin friction is reduced over most of the surface.

Other DNSs considered the flow over triangular riblets. Goldstein, Handler \& Sirovich (1995) emphasized the role of the lateral motion of the turbulence producing eddies and showed that the edges of the riblets have the further beneficial effect of preventing undulations of the near-wall vortical structures, thus providing further insight into the drag-reduction mechanism. The formation of intense vortex structures near the edges of riblets increases the energy dissipation rate and leads to a smaller root mean square (r.m.s.) velocity. Chu \& Karniadakis (1993) found that a local flow reversal occurs within the riblet valleys. However, this is not relevant to the mechanism of drag reduction and was not observed in the two previously mentioned simulations. As for the laboratory experiments of Vukoslavcevic, Wallace \& Balint (1992), the 
mean velocity and Reynolds shear stress profiles were evaluated at three different lateral positions between riblets. Above both the valleys and peaks, there was a reduction relative to the smooth wall channel of the peak r.m.s. value, normalized by the local friction velocity, for both lateral and wall-normal velocity components. The longitudinal Reynolds stress was not reduced, but the location of the peak magnitude of all three normal stresses was shifted well away from the wall.

The present paper represents a first attempt at providing a unified description of the effect that these two different types of roughness may have on the flow. The riblets are treated as a rough surface and, as in the case of the transverse elements, the statistics are evaluated only in the region above the plane of the crests. In contrast to Choi et al. (1993), we do not use a virtual origin for longitudinal elements. Note that previous DNSs have dealt only with riblets of triangular shape, because these are supposedly the most efficient (Walsh 1990). Here, riblets of circular and square cross-sections are also examined. Since our interest is not in maximizing the dragreduction, but in comparing drag-reducing and drag-increasing geometries, the same shape and value of $w / k$ are kept for the two types of roughness. We consider circular, square and triangular bars with $w / k=1$, for which the drag is close to that for a smooth-wall channel.

The mean turbulent kinetic energy production and dissipation rates vary for the two different types of disturbance, owing to changes in the flow structures. To understand whether this variation is caused by large or small scales, profiles of statistics contributing to the energy production are investigated. In order to have a better appreciation of the production mechanism, the energy production term, which is usually written as the product of the Reynolds shear stress and mean velocity gradient, can be expressed in a different way via velocity-vorticity correlations, as suggested by Hinze (1975). The Joint probability density functions (JPDFs) of these correlations are associated with ejection and sweep events, which makes it possible to assess how the structures are modified by different types of roughness. The trace of the velocity-vorticity correlation tensor is linked to the helicity density, which has previously been used by Rogers \& Moin (1987) to highlight departures from isotropy near the wall. This quantity can therefore supplement two-point correlations and anisotropy invariant maps (Leonardi et al. 2004) for quantifying departures from isotropy caused by the roughness.

\section{Numerical procedure}

Several DNSs of flows over irregular walls have been performed. To represent the roughness numerically, two methods can be adopted. One is to use a coordinate transformation where a coordinate line coincides with the wall surface, e.g. Choi et al. (1993). This method requires a large amount of computational time and can be applied only to certain simple surfaces. Since the coordinate is obtained via conformal transformations, this type of study is restricted to certain surfaces (Orlandi 1989). For example, this method cannot handle the flow above circular shaped riblets. Another method is to represent roughness elements by an immersed boundary method as in Goldstein et al. (1995) and Bhaganagar, Kim \& Coleman (2004). This method can handle the flow above any kind of surface, albeit by clustering a larger number of points near the elements.

Since the aim of our paper is to compare roughness elements which are either aligned or placed transversely to the flow direction, the latter method is employed. Details of how to solve the Navier-Stokes equations in a rectangular grid with 
non-uniform spacing in the non-homogeneous direction and uniform spacing in the homogeneous directions can be found in Orlandi (2000). The immersed boundary method in this numerical scheme is described in Fadlun et al. (2000); here, only the main features are recalled. The incompressible non-dimensional Navier-Stokes and continuity equations are

$$
\frac{\partial U_{i}}{\partial t}+\frac{\partial U_{i} U_{j}}{\partial x_{j}}=-\frac{\partial P}{\partial x_{i}}+\Pi \delta_{i 1}+\frac{1}{R e} \frac{\partial^{2} U_{i}}{\partial x_{j}^{2}}, \quad \frac{\partial U_{i}}{\partial x_{i}}=0,
$$

where $R e=\left(U_{c} h / v\right)$ is the Reynolds number, $U_{c}$ is the centreline laminar Poiseuille velocity, $h$ is the channel half-width, $\Pi$ is the pressure gradient required to maintain a constant flow rate, $U_{i}$ is the component of the velocity vector in the $i$ direction, $P$ is the pressure, $x_{1}, x_{2}$ and $x_{3}$ are the streamwise, wall-normal and spanwise directions, respectively. To understand why $U_{c}$ is considered as a reference velocity for turbulent flows, we would like to recall that the simulations start from a laminar flow at $R e=4200$ with a strong random disturbance of zero mean; since the flow rate is constant, the bulk velocity $U_{b}$ is equal to $0.66 U_{c}$. The Navier-Stokes equations have been discretized in an orthogonal coordinate system using a staggered central secondorder finite-difference approximation. In the inviscid case with free-slip conditions, energy is conserved. The discretized system is advanced in time using a fractional-step method with viscous terms treated implicitly and convective terms explicitly. The large sparse matrix resulting from the implicit terms is inverted by performing fast Fourier transforms (FFTs) in the homogeneous directions and applying tridiagonal solvers in the non-homogeneous directions. The reduced wavenumber is used instead of the real wavenumber in order to maintain second-order accuracy of staggered finite differences.

At each time step, the momentum equations are advanced using the pressure at the previous step, yielding an intermediate non-solenoidal velocity field. A scalar quantity $\Phi$ projects the non-solenoidal field onto a solenoidal one. A hybrid low-storage third-order Runge-Kutta scheme is used to advance the equations in time.

The immersed boundary method, used here to describe roughness elements, is similar to that described by Fadlun et al. (2000). This procedure has been applied to a large number of flows, as described in the review of Iaccarino \& Verzicco (2003). As in Fadlun et al. (2000), the present method assumes that the velocities are zero inside the body. However, in Fadlun et al. (2000), the velocity at the first point outside the body is calculated by a linear interpolation with the velocity at the second point outside the body. Here, at the first point outside the roughness element, the discretization of the viscous derivatives in the Navier-Stokes equation is done by taking into account the real distance between the grid point and the walls. To be more specific, the same procedure as for the smooth wall channel is used, but here the metrics vary and depend on the type of surface; near the solid boundary, the scheme is only first-order accurate. This does not disrupt the flow since the flow physics implies that very near a solid boundary, the velocity tends to zero linearly. At a later stage, the accuracy of the method will be ascertained by comparison with experimental results. This treatment of the flow is rather similar to that of Ye et al. (1999) except that the metrics of the $\Phi$ equation are not corrected because the impermeability condition is sufficient for obtaining the correct pressure distribution on the surface of the body.

The constancy of the grid allows the use of FFTs in the homogeneous directions thus leading to very efficient codes. In contrast to smooth-wall channels, at the same Reynolds number $R e$, it is necessary to increase the number of points to describe 


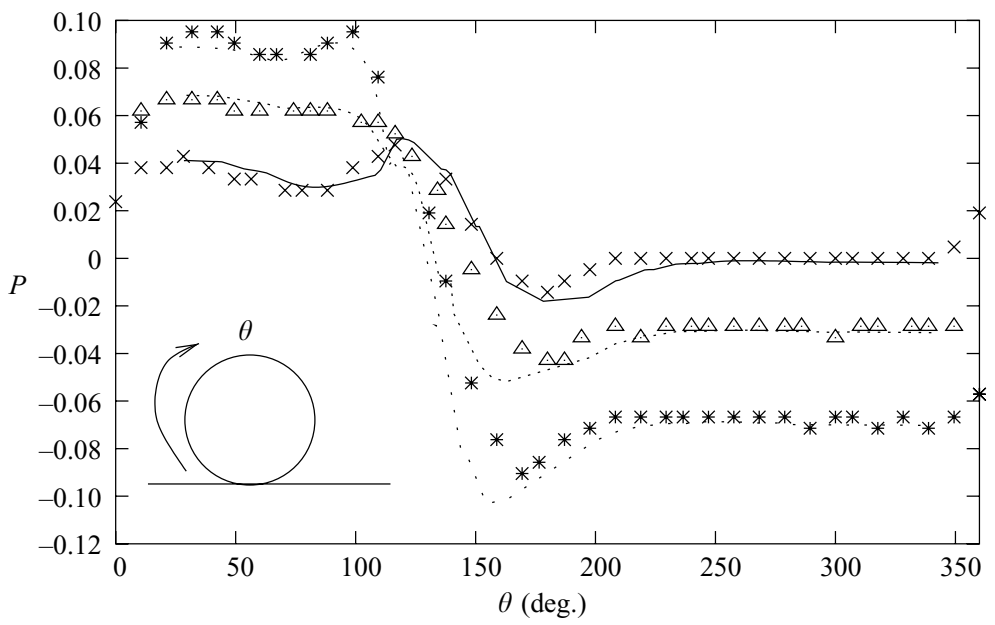

FIGURE 1. Pressure distribution non-dimensionalized by $\rho U_{c}^{2}$ around a circular element, lines are for the present DNS, symbols are for Furuya et al. (1976): -,$\times, w / k=3 ; \cdots \cdots \cdots, . .$, $w / k=7 ; \cdots, *, w / k=15$. The origin of $\theta$ is at the bottom wall, and it is oriented clockwise.

the contour of the body surface accurately. This method is optimal for solving flows in channels where a mean pressure gradient is required to maintain a constant mass flow rate. This mean pressure gradient is evaluated by integrating the streamwise momentum equation.

A validation of the numerical procedure for treating roughness elements is provided by a comparison with the experiment by Furuya et al. (1976). These authors studied the boundary layer over two-dimensional circular rods, fixed to the wall transversely to the flow, for several values of $w / k$ (in this case $w$ is the minimum distance between two rods and $k$ the diameter of the rods). Figure 1 shows that the pressure distributions on the rods are in good agreement with measurements (Furuya et al. 1976). It is important to recall that the latter experiment was carried out in a boundary layer at a larger Reynolds number. A first important inference can be drawn from the agreement in figure 1 . If the main interest is on the near-wall region, there appears to be a similarity between boundary-layer and channel flows, as has previously been established for a smooth surface. For a rough wall, the effect of the Reynolds number is likely to be less critical than for a smooth wall, given that the flow is in the fully rough regime and the major contribution to the wall shear is provided by the form drag of the roughness elements.

This paper considers circular, square and triangular elements, which are either aligned or placed transversely to the flow direction. For ease of identification, the different shapes are denoted by capital letters, $C$ for the smooth-wall channel, $S$ for square bars, $R$ for circular rods and $T$ for triangular elements. The orientation of the elements is indicated by the subscript $N$ for transverse and $P$ for longitudinal elements. The first number in the subscript is the first decimal of the magnitude of $k / h$ and the last is the value of $w / k$ (e.g. $R_{2 N 1}$ corresponds to $k / h=0.2$ and $w / k=1$ ). Statistics for $C, S_{2 N 7}, S_{2 N 1}, R_{2 N 1}, T_{2 N 1}, T_{2 N 0}, S_{2 P 1}, R_{2 P 1}, T_{2 P 1}, T_{2 P 0}, T_{1 P 0}$ are considered. A sketch of the different geometries is shown in figure 2, the origins for the different coordinates used are also included. Although the main focus is on small values of $w / k, S_{2 N 7}$ has been included as a reference case because the roughness function is maximum for this geometry (from Leonardi et al. 2003a). 


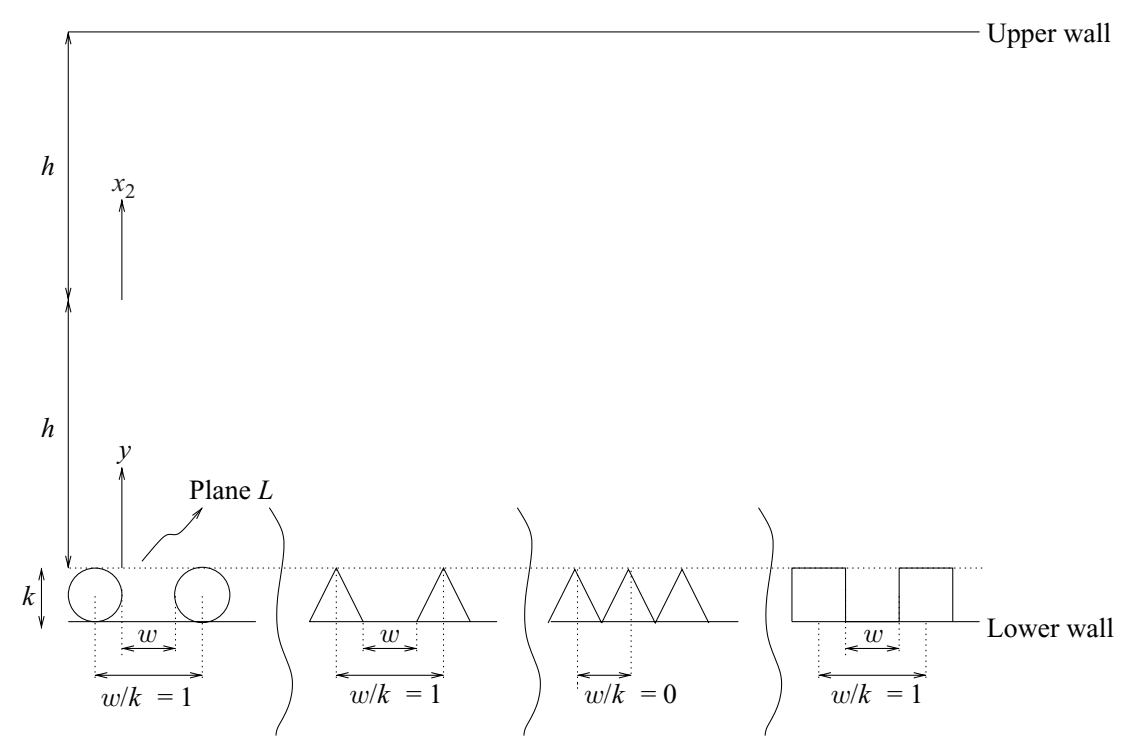

FIGURE 2. Geometrical sketch of the roughness surfaces considered $(\lambda=w+k)$.

For each case, the initial condition is the flow field in a smooth-wall channel at $R e=4200$. Even for this latter simulation, the smooth lower wall is described by the immersed boundary method. Two different computational boxes are used for transverse and aligned disturbances. For transverse elements, the physical dimensions in streamwise and spanwise directions are $L_{1}=8 h$ and $L_{3}=\pi h$. The grid is $400 \times 160 \times 128$ in the streamwise $\left(x_{1}\right)$, normal $\left(x_{2}\right)$ and spanwise $\left(x_{3}\right)$ directions, respectively. In the normal direction, 30 grid points, with an almost uniform spacing, were used for the layer with roughness elements over the range $-1.2<x_{2}<-1$. With 130 points non-uniformly distributed in the range $-1<x_{2}<1$, the first grid point is located approximately 1 wall unit above the roughness crests. Along $x_{1}$, each roughness element is discretized using 10 points.

For the aligned disturbances, $L_{1}$ is the same, but $L_{3}$ is equal to $4 h$ so as to have an integer number of elements along $x_{3}$. In the streamwise direction, 256 points were used. In the spanwise direction, the number of points depends on the height of the elements (only one case was done with triangles with $w / k=0$ and $k / h=0.1$ ). The number of points per element was kept constant, equal to 10 and 30 in the $x_{3}$ and $x_{2}$ directions, respectively. Except for one simulation $(k / h=0.1$ with a grid of $256 \times 160 \times 400$ ), the grid was $256 \times 160 \times 200$. In the following sections, results are presented using normalization based on either wall variables $\left(u_{\tau}, v\right)$ or outer variables $\left(U_{c}, h\right)$. A ' + ' is used in the former case; note that for a rough wall, $u_{\tau}$ has contributions from both the viscous and the form drag.

\section{Mean velocity profiles}

Averages have been performed with respect to $x_{1}$ and $x_{3}$ since our interest is not to account for variations within one roughness wavelength, but to focus on the overall effect the roughness has on the overlying flow. The velocity $U_{i}$ can be expressed as $U_{i}=\left\langle U_{i}\right\rangle+u_{i}^{\prime}$. Figure 3(a) shows that, for disturbances associated with transverse elements, the mean velocity profiles are modified significantly, particularly in the outer region. The maximum is shifted well away from the wall by an amount that depends 

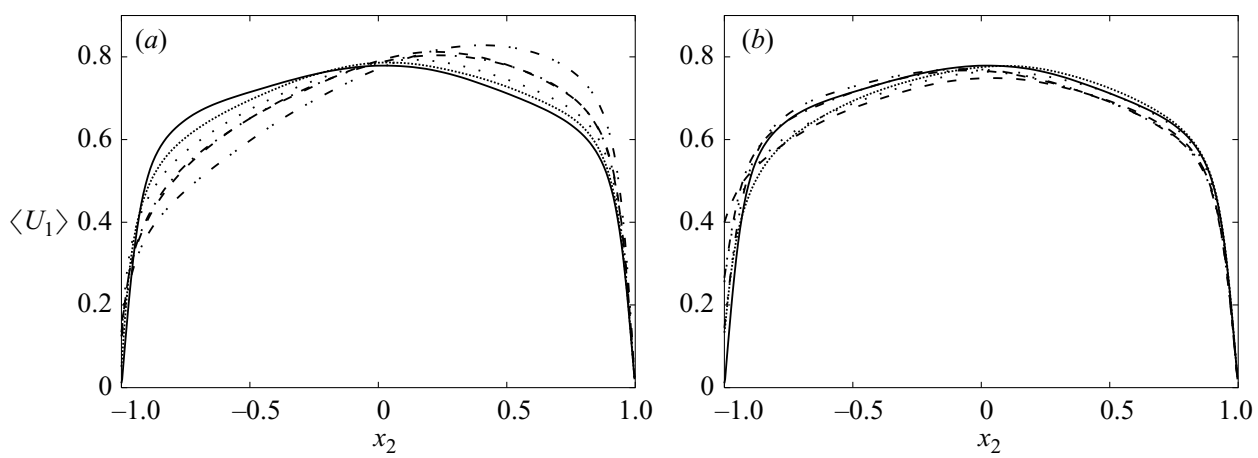

FiguRE 3. Mean velocity profiles. (a) Transverse elements:,$- C ; \cdots \cdots \cdots \cdots, S_{2 N 1} ; \cdots, R_{2 N 1}$; $--\ldots, T_{2 N 0} ;---, T_{2 N 1} ;-\cdots-, S_{2 N 7} .(b)$ Longitudinal elements;,$- C ; \cdots \cdots \cdots, S_{2 P 1}$; $\cdots, R_{2 P 1} ;--, T_{2 P 0} ;---, T_{2 P 1} ;-\cdots-, T_{1 P 0}$.

on the intensity of the disturbance. The relatively large shift towards the outer wall would suggest that the friction on the upper smooth wall increases. However, the increase is not significant; the friction velocity $\left(u_{\tau}\right)_{U}$ is 0.0420 for the smooth wall, and 0.0487 for $S_{2 N 7}$ (the subscript $U$ refers to the upper wall and $u_{\tau}$ is normalized by $U_{c}$ ). At this low Reynolds number, relatively large disturbances can propagate from the lower rough wall to nearly the upper smooth wall. From the minor variations of $\left(u_{\tau}\right)_{U}$, we can infer that, in the near-wall region of a smooth-wall channel, the mechanism of producing and sustaining turbulence is insensitive to relatively small disturbances generated in the outer layer. Clearly, if the disturbances are very large, for example those generated by large-eddy break-up (LEBU) devices, this conclusion would not hold. These devices were studied to investigate the possibility of achieving drag reduction.

The profiles in figure $3(b)$ reveal, on the contrary, large modifications in the region close to the roughness. These are mainly due to the different amounts of area, within the roughness crests plane, where $U_{1}$ can differ from zero. The global effect is equivalent to that obtained by translating the wall in the same direction as the external flow; this motion reduces the velocity gradient at the wall and in the near-wall region. The simulations of Perot \& Moin (1995) and Orlandi \& Leonardi (2001), as well as the experiment by Uzkan \& Reynolds (1967), indicated that this reduces the production of turbulent energy and leads to a drag reduction. The difference between the present longitudinal disturbances and the translating wall is that, in the former case, there is a fluctuating Reynolds shear stress $\left\langle u_{1}^{\prime} u_{2}^{\prime}\right\rangle$ which contributes to the wall shear. Figure $3(b)$ shows that the shift of the maximum towards the rough wall occurs only for the drag reducing cases (viz. $T_{2 P 0}$ and $T_{1 P 0}$ for which $\left(u_{\tau}\right)_{L}$ is 0.0376 and 0.0370 respectively). The subscript $L$ refers to the plane of the crests.

To analyse in more detail the influence of the slip condition, it is useful to plot the profiles of the skin frictional drag. In figures $4(a)$ and $5(a)$, these are normalized with the wall stress for the smooth wall to assess the variations produced by the roughness. The semi-log format emphasizes the region close to the plane of the crests. Hereinafter, $y$ indicates the distance from the plane of the crests $\left(y=1+x_{2}\right.$, for $\left.x_{2}>-1\right)$. This sort of slip condition, for all cases except $S_{2 N 1}$, reduces the velocity gradient at $y=0$ so that the viscous drag is smaller than for the smooth wall. Figure 5(a) shows that when the height of the disturbance is small $(k=0.1)$, the slip velocity is rather small and the velocity gradient is large, although smaller than for the 

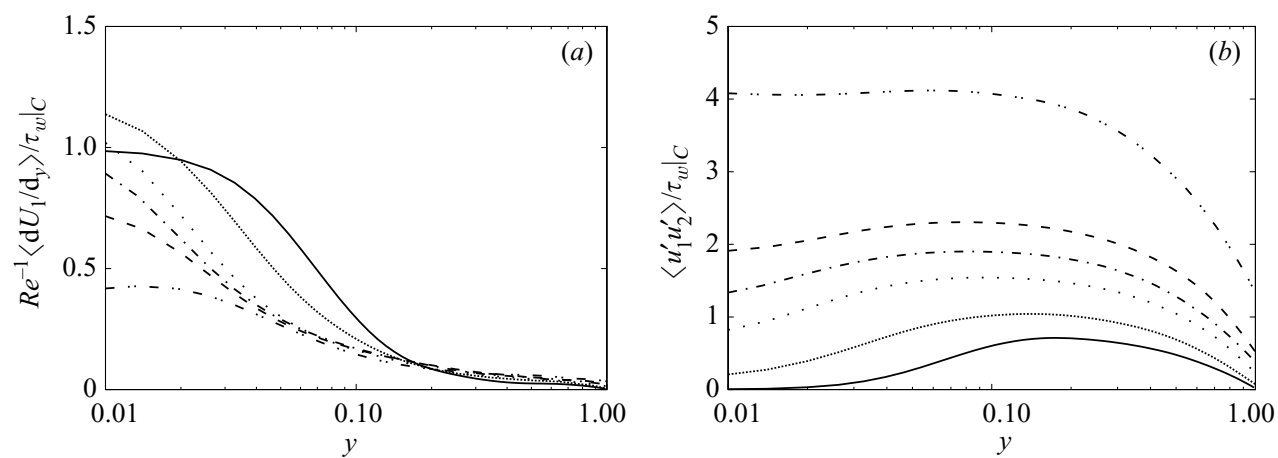

Figure 4. Viscous shear stress $\left(R e^{-1} \mathrm{~d} U_{1} / \mathrm{d} y\right)$ and Reynolds shear stress $\left\langle u_{1}^{\prime} u_{2}^{\prime}\right\rangle$ profiles normalized with $\left.\tau_{w}\right|_{C}$, for transverse elements: -,$C ; \cdots \cdots \cdots \cdots, S_{2 N 1} ; \cdots, R_{2 N 1} ;-\cdots$, $T_{2 N 0} ;---, T_{2 N 1} ;-\cdots-, S_{2 N 7}$.
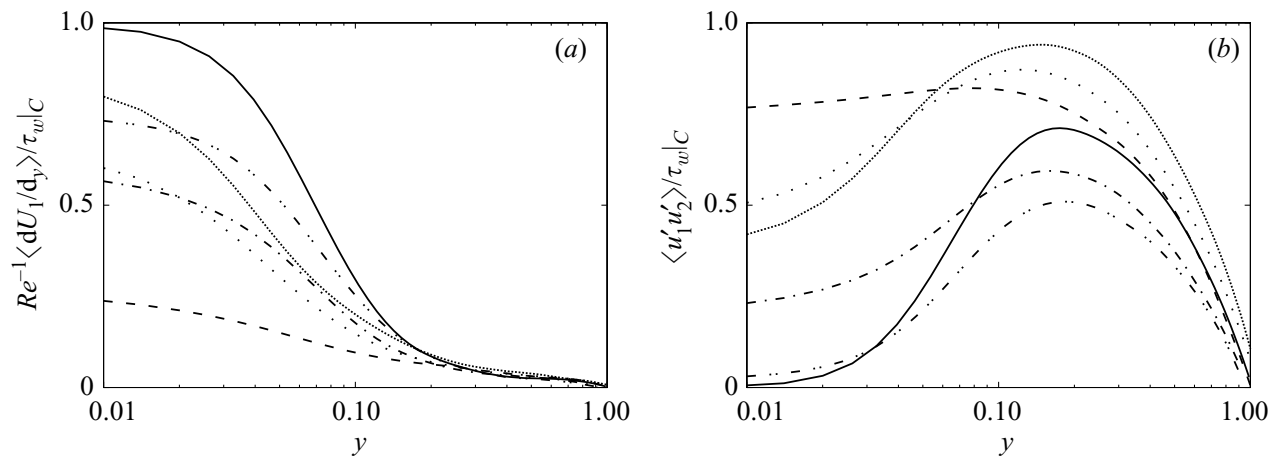

FIGURE 5. Viscous shear stress $\left(R e^{-1} \mathrm{~d} U_{1} / \mathrm{d} y\right)$ and Reynolds shear stress $\left\langle u_{1}^{\prime} u_{2}^{\prime}\right\rangle$ profiles normalized with $\left.\tau_{w}\right|_{C}$, for parallel elements: -,$C ; \cdots \cdots \cdots, S_{2 P 1} ; \cdots, R_{2 P 1} ;--, T_{2 P 0}$; ,$--- T_{2 P 1} ;-\cdots-, T_{1 P 0}$.

smooth-wall channel. For triangles with $k=0.2$ and $w / k=1$, the slip velocity is large and hence the viscous friction is rather small. As expected, figure 4(a) shows that, for the transverse elements, the minimum viscous stress is obtained for $S_{2 N 7}$, where the relatively large separation between the elements produces a quite large $\left\langle U_{1}\right\rangle$ at the plane of the crests. To estimate the total drag over the rough wall, the Reynolds shear stress should be added to the viscous stress. For transverse roughness, the Reynolds shear stress is proportional to the form drag (Leonardi et al. 2003a), and figure 4(b) shows that, if the disturbance is large $\left(S_{2 N 7}\right)$, the shear stress $\left\langle u_{1}^{\prime} u_{2}^{\prime}\right\rangle$ can be four times as large as the maximum shear stress for the smooth-wall channel. Despite the reduction of the viscous stress for this case, $\left(u_{\tau}\right)_{L}$ reaches a value of 0.0897 , i.e. more than twice that of case $C$, where $\left(u_{\tau}\right)_{L}$ is 0.0423 . Except for $R_{2 N 1}$ and $S_{2 N 1}$, the form drag dominates the frictional drag. For triangular elements, the viscous stresses are smaller than for the smooth wall, but the form drag is large.

For the flow-aligned elements, figure 5(a) shows that, at the same value of $w / k$, the viscous contribution decreases as the area, without a solid surface, at the plane of the crests increases. In this area, a quasi-free-slip condition applies. The opposite occurs for the Reynolds shear stress (figure $5 b)$, and, as a result, $\left(u_{\tau}\right)_{L}$ increases, $(0.0463$, 0.0452 and 0.0425 for $S_{2 P 1}, R_{2 P 1}$ and $\left.T_{2 P 1}\right)$. These latter values do not differ appreciably 

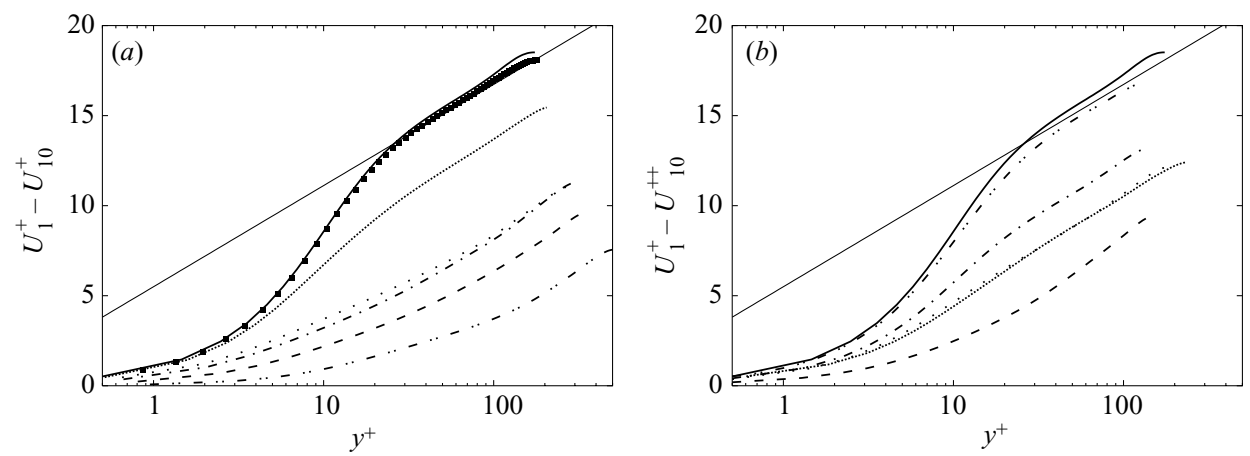

FiguRE 6. Velocity profiles in wall units. (a) Transverse elements:,$- C ; \cdots \cdots \cdots . . ., S_{2 N 1}$; $\cdots, R_{2 N 1} ;---, T_{2 N 0} ;---, T_{2 N 1} ;-\cdots-, S_{2 N 7} .(b)$ Parallel elements:,$- C ; \cdots \cdots \cdots \cdots$, $S_{2 P 1} ; \cdots, R_{2 P 1} ;--T_{2 P 0},---, T_{2 P 1} ;-\cdots-, T_{1 P 0}$. In $(a)$ the closed squares represent the data of Kim et al. (1987) and the straight line is $U^{+}=1 / 0.41 \ln \left(y^{+}\right)+5.5$.

from the smooth-wall friction velocity value. On the other hand, for the triangles with $w / k=0$, drag reduction is achieved for $T_{1 P 0}$, and this is obtained via a relatively high viscous stress, though smaller than that for a smooth wall, and a small $\left\langle u_{1}^{\prime} u_{2}^{\prime}\right\rangle$. The resulting value of $\left(u_{\tau}\right)_{L}$ is 0.0370 which is comparable to $\left(u_{\tau}\right)_{L}=0.0376$ for $T_{2 P 0}$. Since the simulation $T_{2 P 0}$ contradicts the results of Walsh (1990), the grid was refined. A very coarse simulation with $129 \times 101$ points in $x_{1}$ and $x_{3}$, respectively, yielded $\left(u_{\tau}\right)_{U}=0.0585$ and $\left(u_{\tau}\right)_{L}=0.0440$. A finer grid, $(129 \times 201)$ yielded $\left(u_{\tau}\right)_{U}=0.0419$ and $\left(u_{\tau}\right)_{L}=0.0388$. The values $\left(u_{\tau}\right)_{U}=0.0402$ and $\left(u_{\tau}\right)_{L}=0.0370$, obtained with the finest grid, are expected to be grid independent.

In the near-wall region, figures $4(b)$ and $5(b)$ show that the Reynolds shear stress $\left\langle u_{1}^{\prime} u_{2}^{\prime}\right\rangle$, in some of the cases and in particular those with large disturbances, is approximately constant. This behaviour could help experimentalists to infer the wall shear stress from values of $\left\langle u_{1}^{\prime} u_{2}^{\prime}\right\rangle$ that are measured some distance from the wall.

The effective origin for $y$ in (1.1) is at a distance $d_{0}$ from the roughness crests plane; this 'error in origin', can be determined in several different ways. For example, Jackson (1981) evaluated $d_{0}$ as the centroid of the moment of forces around the roughness element. In experiments, $d_{0}$ is often calculated by assuming the validity of the log-law with a value of 0.41 for the Kármán constant $\kappa$. To compare with experiments, Leonardi et al. (2003a), also made this assumption. Here, $d_{0}$ coincides with the plane of the roughness crests $\left(x_{2}=-1\right)$. At this location, depending on the type of disturbance, the mean velocity is $U_{10}=\left\langle U_{1}\right\rangle_{L} \neq 0$. It follows that, relative to this origin, the mean velocity, normalized by wall variables, is $U^{+}=\left(\left\langle U_{1}\right\rangle-U_{10}\right) /\left(u_{\tau}\right)_{L}$. We recall that the friction velocity, $\left(u_{\tau}\right)_{L}$, is given by the square root of the sum of the skin frictional drag $\tau_{f}=\left(R e^{-1} \partial\left\langle U_{1}\right\rangle / \partial y\right)_{L}$ and the form drag whereas for a smooth wall, only the skin friction contributes to $u_{\tau}$. On each side of the channel, $\left\langle U_{1}\right\rangle$ is normalized by the appropriate $u_{\tau}$ from the wall up to $y_{\max }$, the location of the maximum streamwise velocity. The profiles on the smooth-wall side of the channel are not reported, but they overlap and differ only in terms of the extent of the log-law region.

Figure 6 shows mean velocity profiles normalized by wall variables. The thick line for the smooth-wall channel is given by $U^{+}=(1 / 0.41) \ln y^{+}+5.5$ and this agrees reasonably well with the velocity profile at $R_{\tau}=u_{\tau} h / v=180$ by Kim, Moin \& Moser 


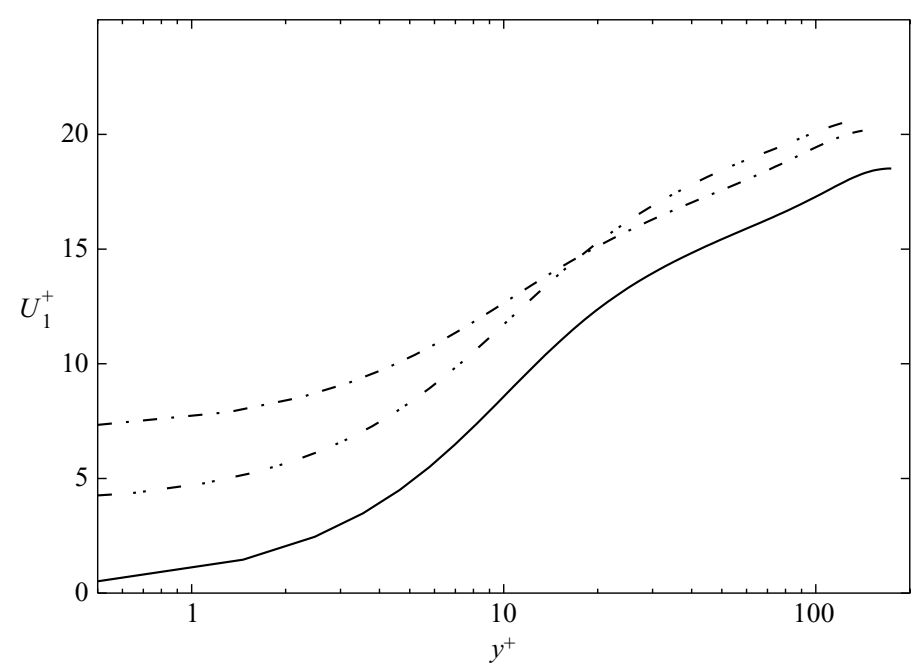

FIGURE 7. Velocity profiles in wall units without subtracting $U_{10}^{+}$as in Choi et al. (1993): ,$- C ;--, T_{2 P 0} ;-\cdots-, T_{1 P 0}$.

(1987); the small difference between the present results and those by Kim et al. (1987) is probably caused by the width $L_{3}$ of the channel, here assumed equal to $\pi$ instead of $2 \pi$. To evaluate the shift with respect to the smooth wall, a log-law, tangent to each profile, can be evaluated with a value of 0.41 for $\kappa$. It can be appreciated that, to a close approximation, a reasonable log-region appears in each case. The other interesting outcome is that, at this low $R e$, by increasing the effect of the disturbance $\left(T_{2 N 0}, T_{2 N 1}, S_{2 N 7}\right)$, the extent of the 'wake' region increases and a distinction between viscous and buffer regions is no longer discernible. The tangent lines are not reported in figure 6, but these were used to evaluate the roughness function. For flow-aligned disturbances, a similar behaviour is observed, and it turns out that also for the drag reducing geometries $\left(T_{2 P 0}, T_{1 P 0}\right)$ there is a downward shift of the log-law, which seems to contradict what Choi et al. (1993) observed. In that paper, the effect of the virtual origin $\left(y_{0}\right)$ on the upward shift was investigated and when $y_{0}$ coincided with the tip of the riblets, the maximum shift was found. The downward shift can therefore be attributed to the subtraction of $U_{10}^{+}$. This quantity should in fact be added to obtain $U_{1}^{+}$, as was done by Choi et al. (1993), and to show the upward shift for drag-reducing riblets. The more conventional plot of figure 7 clearly shows that drag reduction is achieved for $T_{2 P 0}$ and $T_{1 P 0}$. The two curves cross and although $U_{10}^{+}$is greater for $T_{2 P 0}$ than $T_{1 P 0}$, the drag reduction is smaller in the former case.

From the data in figure 6, the roughness function $\Delta U^{+}$can be evaluated and plotted versus $w / k$; the trend is the same as that of Furuya et al. (1976) for circular rods, Krogstad \& Antonia (1999) and Moore (1951) for square bars, and the DNS results of Leonardi et al. (2003a). By plotting these data as a function of $w / k$ it appears that the shape of the geometry plays an important role for small $w / k$ and that the effect is reduced for $w / k>3$. Whereas $U^{+}$is given by $\left\langle U_{1}\right\rangle / u_{\tau}$ in experiments, here $U^{+}=\left(\left(\left\langle U_{1}\right\rangle-U_{10}\right) / u_{\tau}\right)_{L}$, so that $U^{+}$is shifted downward by a factor $\left(U_{10} / u_{\tau}\right)_{L}$. This procedure is not possible in the laboratory because $U_{10}$ cannot be easily measured; Leonardi et al. (2003a) followed the experimental procedure for validating the simulations. This is why the present roughness function is larger than that in Leonardi et al. (2003a) which was obtained with the same database. 


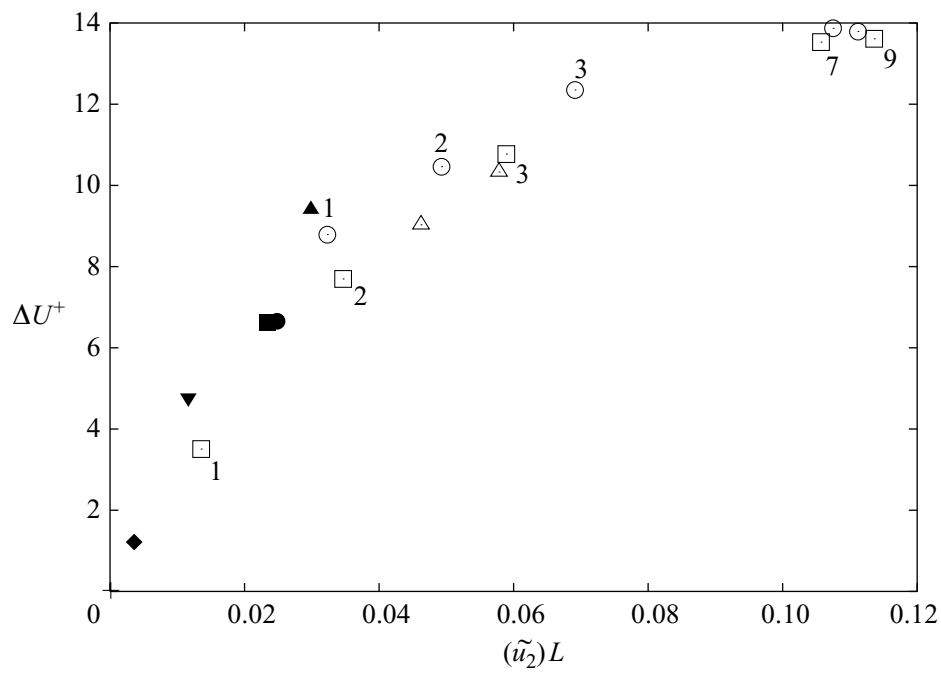

FiguRE 8. Roughness function $\left(\Delta U^{+}\right)$versus $\widetilde{u_{2}}$ at the roughness crests plane, open symbols transverse elements: $\bigcirc$, rods $(w / k=1,2,3,7,9) ; \square$, bars $(w / k=1,2,3,7,9) ; \Delta$, triangles $(w / k=0,1)$; closed symbols longitudinal elements: $\bullet, R_{2 P 1} ; \boldsymbol{\square}, S_{2 P 1} ; \boldsymbol{\Lambda}, T_{2 P 1} ; \nabla, T_{2 P 0} ; \bullet, T_{1 P 0}$.

However, once a decision on how to normalize the profiles has been made, data for different geometries do not collapse when $w / k$ is varied. We can speculate that even by choosing different geometrical parameters, for example the ratio between solid and fluid spaces, a collapse is improbable. In particular, a collapse of the data is impossible when the ratio between solid and empty spaces is approximately 1 . This applies typically to practical situations, such as flows over urban areas or flows in heat exchangers. As mentioned previously, a parameter which relates to the flow statistics should lead to a better collapse of the data. Indeed, figure 8 shows that, by plotting the roughness function against $\left(\widetilde{u_{2}}\right)_{L}=\left\langle u_{2}^{\prime 2}\right\rangle_{L}^{1 / 2}$ (a tilde will be used to denote a r.m.s. value), a better collapse of the data is achieved. This is consistent with the analysis of Orlandi et al. (2003). In addition, figure 8 shows that the same scaling holds for the longitudinal elements. This is a clear indication that the driving mechanism of the near-wall vortical structures is related to the normal velocity fluctuations in the plane of the crests. One could then design the disturbance so as to increase $\left(\widetilde{u_{2}}\right)_{L}$ if the objective were to increase the drag. As pointed out by Belcher, Jerram \& Hunt (2003), geometrical parameters are not sufficient for classifying the large variety of roughnesses. They claim that better modelling is needed, particularly for the non-uniform regions in the vicinity of the roughness, in order to tackle practical problems such as mesoscale numerical weather prediction and air quality forecasting in urban areas and hilly terrains. The validity of this better parameterization is limited, at the moment, to two-dimensional disturbances at low Reynolds numbers. Simulations at high Reynolds numbers and with three-dimensional surfaces have not yet been performed; the extension to three-dimensional roughness is currently being pursued.

\section{Turbulent stresses}

As expected, turbulent intensities can differ significantly, depending on the type of disturbance. For transverse elements, the roughness effects should penetrate further 

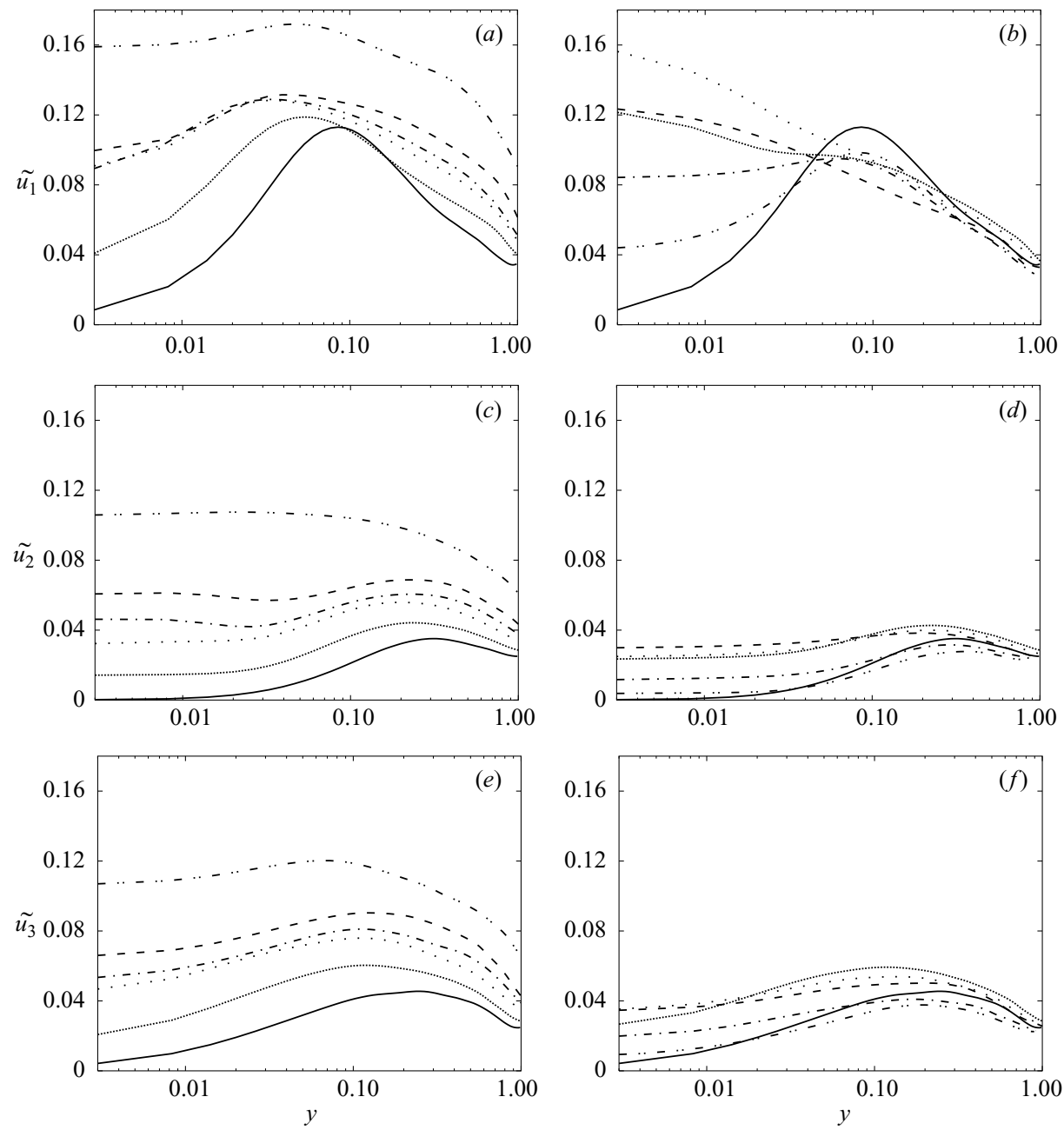

FIgURE 9. Turbulent intensities profiles: $(a, b)$ streamwise, $(c, d)$ normal, $(e, f)$ spanwise; $(a, c, e)$ transverse elements:,$- C ; \cdots, S_{2 N 1} ; \cdots, R_{2 N 1} ;-\cdots, T_{2 N 0} ;---, T_{2 N 1}$; $-\cdots-, S_{2 N 7} .(b, d, f)$; longitudinal elements,,$- C ; \cdots \cdots \cdots, S_{2 P 1} ; \cdots, R_{2 P 1} ;-\cdots$, $T_{2 P 0} ;---, T_{2 P 1} ;-\cdots-, T_{1 P 0}$.

into the flow. Both classes of disturbances are expected to affect profiles near the roughness crests plane significantly. To emphasize differences between different stresses, the same scale is used for the ordinates. The normalization is with respect to $U_{c}$ in order to highlight the large variations that occur. Figures $9(a)$ and $9(b)$ show that $\widetilde{u_{1}}$ exhibits very large variations, for both kinds of disturbance, very near the roughness crests plane. The difference is that, for transverse elements, the increase is transferred to the outer region, while, for flow-aligned elements, the increase is limited to the near-wall region. For transverse elements, fluctuations in $u_{1}^{\prime}$ are related to the strength of the recirculating region between elements. For instance, for square bars, Leonardi et al. (2003a) showed that, for $w / k=7$, this recirculation is similar to the large intermittent recirculation region behind a backward-facing step, and hence is capable of generating very large fluctuations at the interface. On the other hand, for the flow-aligned elements, the fluctuations in $u_{1}^{\prime}$ depend on the extent of 
the region associated with a quasi-free-slip condition for the streamwise velocity. For $T_{1 P 0}$, this layer is smaller than in the other cases and the profile resembles that of the smooth-wall channel. The well-defined peak observed for this case is absent in other geometries. With regard to the penetration of the disturbance, the profiles (not shown here) near the opposite smooth wall, show that there is a slight increase of $\widetilde{u_{1}}$ for $S_{2 N 7}$. However, when the normalization is on wall variables, this increase is no longer apparent and thus the profiles coincide with case $C$. This collapse further confirms that, for smooth walls, there is a quasi-universal wall scaling, irrespective of any kind of disturbance originating in the outer layer.

The penetration is related to the intensity of $\left(\widetilde{u_{2}}\right)_{L}$ which, according to figure $9(c)$, increases systematically for the transverse elements. The increase is reduced for the flow-aligned elements (figure $9 d$ ). The difference is related to the different nature of the recirculation zone between the elements. For disturbances orthogonal to the flow, there are separation and reattachment points, which, for $w / k \approx 1$, are located near the leading and trailing edges of the elements in the plane of the roughness crests. As mentioned above, the nature of the recirculation region is different for $S_{2 N 7}$. For any kind of geometry, the recirculating regions are driven directly by the flow and have the same sense of rotation in every cavity. For longitudinal disturbances, the recirculating regions consist of secondary motions driven by the pressure difference between the two walls; in each cavity, the direction of rotation can change. The secondary motion is weaker than the recirculation associated with the transverse roughness and, as a consequence, $\left(\widetilde{u_{2}}\right)_{L}$ is smaller. Further, by decreasing the height of the elements and the width of the cavities, the strength of the secondary motion decreases. This explains why the smallest $\widetilde{u_{2}}$ occurs for $T_{1 P 0}$. The same considerations hold for $\widetilde{u_{3}}$ (figure $\left.9 e, f\right)$; as for $\widetilde{u_{2}}$, aligned disturbances with the same $w / k\left(S_{2 P 1}\right.$, $\left.R_{2 P 1} T_{2 P 1}\right)$ yield stresses which are larger than those of the smooth-wall channel, both in the outer and near-wall regions.

For the smooth-wall channel, it is well known that $\widetilde{u_{1}}$ and $\widetilde{u_{3}}$ increase with $y$ and $\widetilde{u_{2}}$ with $y^{2}$ in the limit $y \rightarrow 0$. For elements with $w / k \leqslant 1$, the growth rate is reduced, but is not negligible. On the other hand, $\widetilde{u_{2}}$ is almost constant in the layer very near the roughness crests plane. As discussed previously, this could be important in the laboratory to avoid making measurements very near the plane of the crests. Such measurements are in fact, necessary to check whether the proposed new scaling for the roughness function (figure 8) is valid at higher Reynolds-numbers, and it is well recognized that the Reynolds-number dependence can be more easily demonstrated by measurement than with the use of DNS data. In addition, figure $9(c)$ shows that, when the disturbance is strong, $\widetilde{u_{2}}$ decreases more rapidly than for weak disturbances far from the wall; this was explained by Leonardi et al. (2003b) through the transport equation for the wall-normal Reynolds stress, the production rate of $\widetilde{u_{2}}$ diminishing more rapidly for $w / k=7$ than for $w / k=3$. A similar reduction occurs for the other two stresses, as shown in figure $9(a)$ and figure $9(e)$.

Leonardi et al. (2004) showed that the square bars enhance the isotropy of the stresses; the streamwise turbulent intensity increases by a smaller amount than the other two normal stresses. Here we extend this finding to triangular and circular elements, with particular emphasis on small $w / k$. To quantify the effect that the orientation of the elements has on the near-wall isotropy, the quantity $q_{i}=\left\langle u_{i}^{\prime 2}\right\rangle / q^{2}$, where $q^{2}\left(\equiv\left\langle u_{1}^{\prime 2}\right\rangle+\left\langle u_{2}^{\prime 2}\right\rangle+\left\langle u_{3}^{\prime 2}\right\rangle\right)$ is twice the turbulent kinetic energy, has been calculated at the first point close to the roughness crests plane. Figure 10 shows that the improved isotropy is quite well correlated with $\left(\widetilde{u_{2}}\right)_{L}$. For small $\left(\widetilde{u_{2}}\right)_{L}$, the difference between $q_{1}$ and $q_{2}$ is large, especially for the flow-aligned elements. For the 


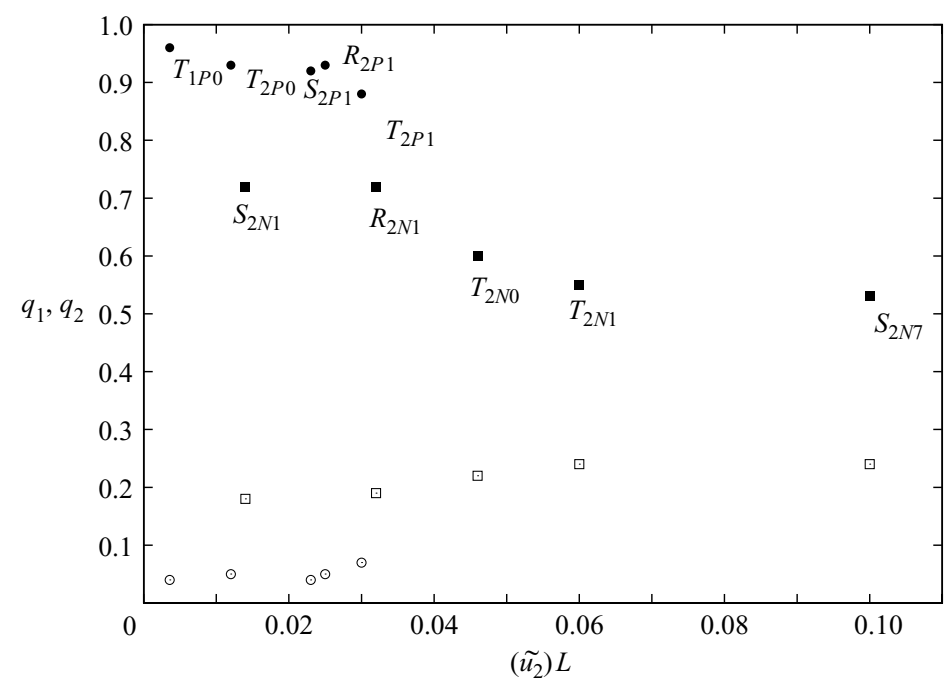

FIGURE 10. Measure of anisotropy at the roughness crests plane. $\square$, transverse elements; $\bigcirc$, flow-aligned elements. Solid symbols, $q_{1}$; open symbols, $q_{2}$.

largest $\left(\widetilde{u_{2}}\right)_{L}$, which corresponds approximately to the maximum drag, the difference between $q_{1}$ and $q_{2}$ is much reduced, consistent with an approach towards isotropy. Since organized wall structures are characterized by a high level of anisotropy, we can infer that a reduced organization leads to an increase in drag.

Root mean square turbulent intensities, normalized by wall variables, are shown in figure 11 . The relatively good agreement with the stresses of Kim et al. (1987) further validates the present numerical method. In agreement with Bhaganagar et al. (2004), the roughness reduces the near-wall maximum of ${\widetilde{u_{1}}}^{+}$with respect to the smooth wall. These authors considered a three-dimensional roughness which should produce a large $\left(\widetilde{u_{2}}\right)_{L}$; the peak value of ${\widetilde{u_{1}}}^{+}$was approximately 1.5 , in good agreement with the present value for $S_{2 N 7}$. The present results also show that the shape of the profiles in the near-wall region depends on the geometry and, when the disturbance is very strong, the peak almost disappears, regardless of which particular r.m.s. intensity is considered. The increase in ${\widetilde{u_{2}}}^{+}$and ${\widetilde{u_{3}}}^{+}$implies that these r.m.s. intensities increase more rapidly than $\left(u_{\tau}\right)_{L}$. This is expected since the shape of the geometry affects mainly $u_{2}^{\prime}$ and $u_{3}^{\prime}$ at the roughness crests plane. From these results, we can infer that, for rough-wall flows, the velocity r.m.s. does not scale in the wall region with the friction velocity. The r.m.s. profiles (not shown here) on the upper wall are not affected up to $y^{+}=40$ by the roughness on the bottom wall. This confirms that turbulence in the near-wall region can be modified only by local disturbances or by disturbances imposed at the wall itself. As mentioned earlier, we are referring only to weak disturbances propagating from the outer layer towards the wall. The profiles in figure $11(b, d, f)$ have the same behaviour as in figure $9(b, d, f)$ This is expected because the friction velocities do not vary significantly among the different flow-aligned elements.

The linear decay of the total stress characterizes fully developed channel and pipe flows; this is also true for rough-wall channels, independently of the shape of the elements. The establishment of a linear profile, not shown here, is a check that the number of fields used for calculating the statistics is adequate. As is well known, the Reynolds shear stress $\left\langle u_{1}^{\prime} u_{2}^{\prime}\right\rangle$ converges much more slowly than the normal stresses. 

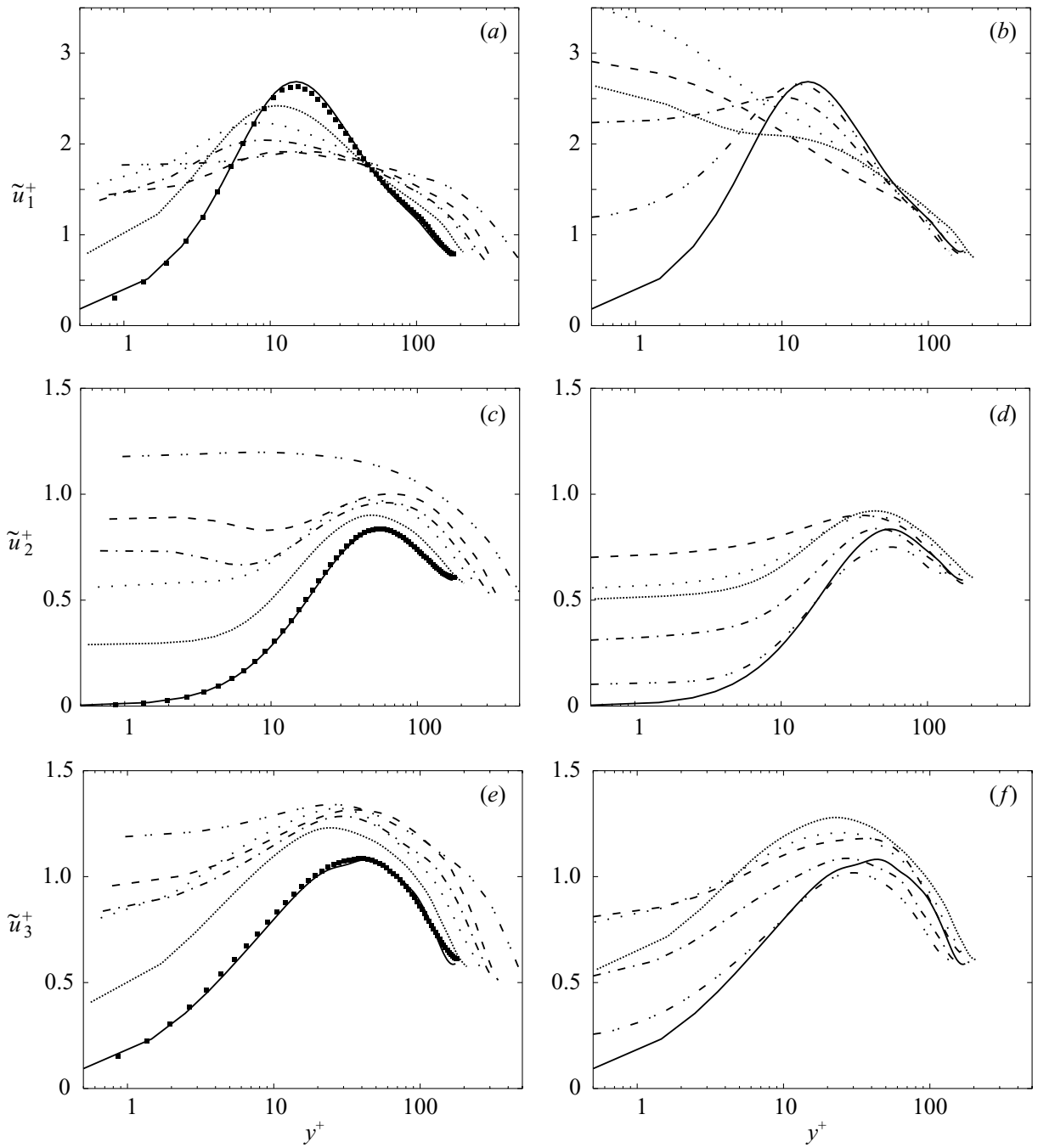

FiguRE 11. Turbulent intensities in wall units: $(a, b)$ streamwise, $(c, d)$ normal, $(e, f)$ spanwise; $(a, c, e)$ transverse elements: -,$C ; \cdots \cdots \cdots, S_{2 N 1} ; \cdots, R_{2 N 1} ;-\cdots, T_{2 N 0} ;---, T_{2 N 1}$; $-\cdots-, S_{2 N 7} ;(b, d, f)$, longitudinal elements:,$- C ; \cdots \cdots \cdots, S_{2 P 1} ; \cdots, R_{2 P 1} ;-\cdots$, $T_{2 P 0} ;---, T_{2 P 1} ;-\cdots-, T_{1 P 0}$. Symbols, Kim et al. (1987).

Relative to the geometrical centreline, the zero crossing point of the total stress is shifted upwards for transverse elements and downwards for the drag-reducing roughness $T_{1 P 0}$ and $T_{2 P 0}$. The shift increases as the disturbance increases. The zero crossing point for $\left\langle u_{1}^{\prime} u_{2}^{\prime}\right\rangle$ coincides with that for $\partial\left\langle U_{1}\right\rangle / \partial x_{2}$ for $w / k=0$ and 1. Leonardi, Orlandi \& Antonia (2005) showed that this coincidence does not apply at larger values of $w / k$.

Near a smooth wall, the viscous stress dominates over the turbulent stress. For rough walls, the stresses can be of the same order depending on the geometry of the roughness. The profiles of the two stresses given in figures 4 and 5 are proportional to the quantities in the expression for the turbulent kinetic energy production $-\left\langle u_{1}^{\prime} u_{2}^{\prime}\right\rangle \partial\left\langle U_{1}\right\rangle / \partial x_{2}$. The large changes lead to a significant modification in the turbulence production mechanism, as described in the following section. 


\section{Turbulence production}

In turbulent wall flows, the kinetic energy is produced mainly at $y^{+} \approx 10$ and then transferred towards the wall, where it is dissipated, and to the interior of the channel. For smooth walls, the energy dissipation rate is essentially balanced by viscous diffusion. Leonardi et al. (2003b) showed that the energy budgets for square bars and circular rods are approximately the same for $w / k=3$ and $w / k=7$. The production is the biggest term across the wall layer and is balanced by the sum of the dissipation rate and the turbulent diffusion. Since there are large modifications to the production term, this term is analysed in more detail here. The expression for the turbulent kinetic energy production can be decomposed as follows

$$
\mathscr{P}=\overbrace{\left\langle U_{1}\right\rangle\left[\left\langle u_{3}^{\prime} \omega_{2}^{\prime}\right\rangle-\left\langle u_{2}^{\prime} \omega_{3}^{\prime}\right\rangle\right]}^{1} \overbrace{\frac{\partial\left\langle U_{1}\right\rangle\left\langle u_{1}^{\prime} u_{2}^{\prime}\right\rangle}{\partial x_{2}}}^{2} .
$$

The derivation of this equation which separates contributions from the active and inactive motions, is given by Hinze (1975, p. 680). The inactive or irrotational motion acts through the pressure field. A new pressure $\mathscr{P}$ can be defined as $\mathscr{P}=p+\left\langle u_{i}^{\prime} u_{i}^{\prime}\right\rangle / 2$. The active motion is given by (5.1) where term $2,-\partial\left\langle U_{1}\right\rangle\left\langle u_{1}^{\prime} u_{2}^{\prime}\right\rangle / \partial x_{2}$, is a redistribution term. For the smooth channel, it integrates to zero. Far from the wall, this term is negative since energy is lost by the large scales, whereas near the wall, energy is gained by the large scales. Term 1 is related to the transfer of energy between large and small scales. Near the wall, energy is lost to the small scales (the sign of term 1 is negative) whereas, in the central region, it is gained from the small scales (the sign of term 1 is positive). The difference between terms 1 and 2 in (5.1) represents the turbulent energy production, which is positive everywhere. Far from the wall, the two terms are in approximate balance.

Here, the interest is only in the active motion responsible for the Reynolds shear stress; we speculate that, in order to achieve a drag increase and hence an increase of turbulent energy production, the cross-product $\boldsymbol{v}^{\prime} \times \omega^{\prime}$ should increase. For drag reduction, this product should decrease. Using the identity

$$
\left|\boldsymbol{v}^{\prime} \times \boldsymbol{\omega}^{\prime}\right|^{2}+\left|\boldsymbol{v}^{\prime} \cdot \boldsymbol{\omega}^{\prime}\right|^{2}=\left|\boldsymbol{v}^{\prime}\right|^{2}\left|\boldsymbol{\omega}^{\prime}\right|^{2},
$$

a drag reducing flow should have a greater alignment between velocity and vorticity vectors. An increase in $\boldsymbol{v}^{\prime} \cdot \boldsymbol{\omega}^{\prime}$ was found by Orlandi (1997) in rotating pipes and the relative drag reduction was explained through an increased organization of the structures. Before examining how the roughness affects term 1 in (5.1), it is worth showing how the contributions behave over a smooth wall. In figure 12, the rotational term (which may be interpreted as a body force term) indicates whether the kinetic energy is either transferred from large to small scales or gained by the large scales. Near the wall, the kinetic energy resides at large scales, of the order of the distance from the wall. Therefore the active motion indicates a transfer of energy to the small scales. In the outer region, the energy containing scales are comparable to the channel size and the rotational term indicates a transfer to large scales. Term 2 of of (5.1) is positive near the wall and greater than the rotational term. The net outcome is a large positive production in the buffer layer. In the outer region, term 2 indicates that approximately the same amount of energy which is fed to the large scales (characteristic of the outer layer) comes from the small scales. The net result is a positive production, smaller than that in the buffer region, which tends to zero near the centreline. 


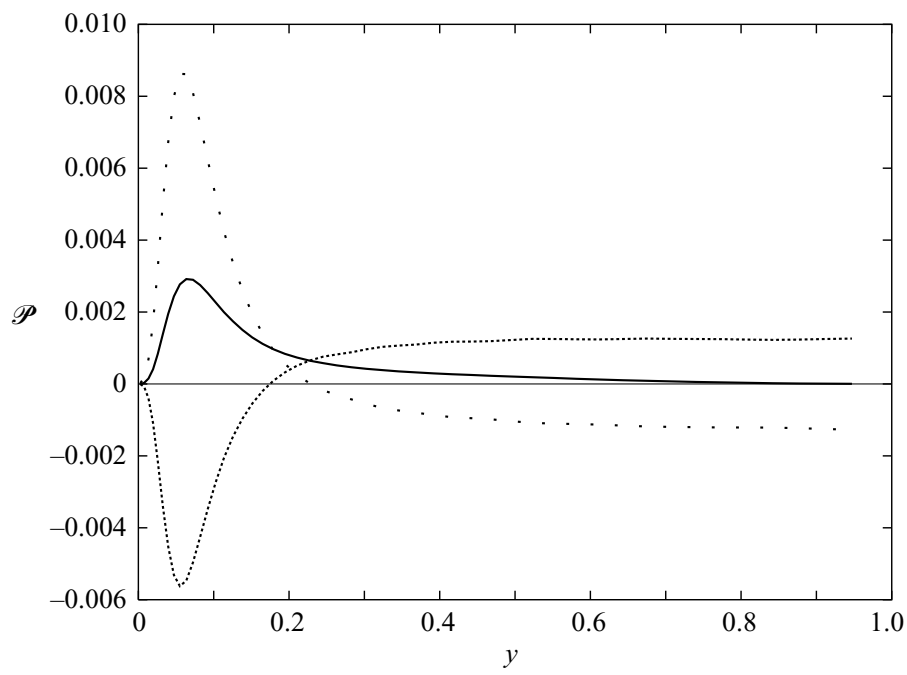

FiguRE 12. Production for the smooth-wall channel (only one side).,$- \mathscr{P}$; ,$\left\langle U_{1}\right\rangle\left[\left\langle u_{3}^{\prime} \omega_{2}^{\prime}\right\rangle-\left\langle u_{2}^{\prime} \omega_{3}^{\prime}\right\rangle\right] ;---, \partial\left\langle U_{1}\right\rangle\left\langle u_{1}^{\prime} u_{2}^{\prime}\right\rangle / \partial x_{2}$ (see $\left.(5.1)\right)$.
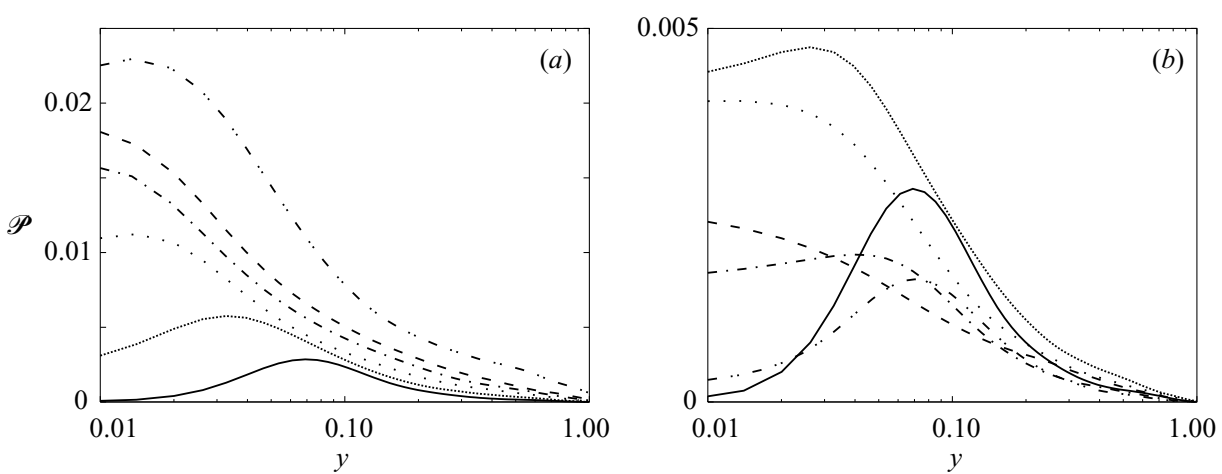

Figure 13. Profiles of turbulent kinetic energy production. (a) Transverse elements: $C ; \cdots \cdots \cdots, S_{2 N 1} ; \cdots, R_{2 N 1} ;---, T_{2 N 0} ;---, T_{2 N 1} ;-\cdots-, S_{2 N 7} .(b)$ Longitudinal elements,$- C ; \cdots \cdots \cdots \cdots, S_{2 P 1} ; \cdots, R_{2 P 1} ;---, T_{2 P 0} ;---, T_{2 P 1} ;-\cdots-, T_{1 P 0}$.

A qualitative explanation for the effects of the roughness on the three normal stresses, indicated in figure 9, can be inferred from the profiles of $\mathscr{P}$ (figure 13). From the distributions in figure 9, it can be deduced that for the transverse elements, $\left\langle q^{2}\right\rangle$ increases across the channel whereas, for longitudinal elements, the increase is restricted to a thin layer close to the roughness crests plane. In the remainder of the channel, $\left\langle q^{2}\right\rangle$ is smaller than over a smooth wall. The profiles of $\mathscr{P}$ in figure 13 reflect to a large extent the changes in $\left\langle q^{2}\right\rangle$. A comparison between figure 13(a) and figure 13(b) highlights that the increase in production near the roughness crests plane is smaller for longitudinal than for transverse elements. For longitudinal elements, the production is reduced at a distance from the wall which corresponds to the maximum production over a smooth wall. The inference is that the energy-containing eddies are smaller near longitudinal elements. For the drag-reducing cases, it seems that the production is reduced because the near-wall vortical structures are more organized. 

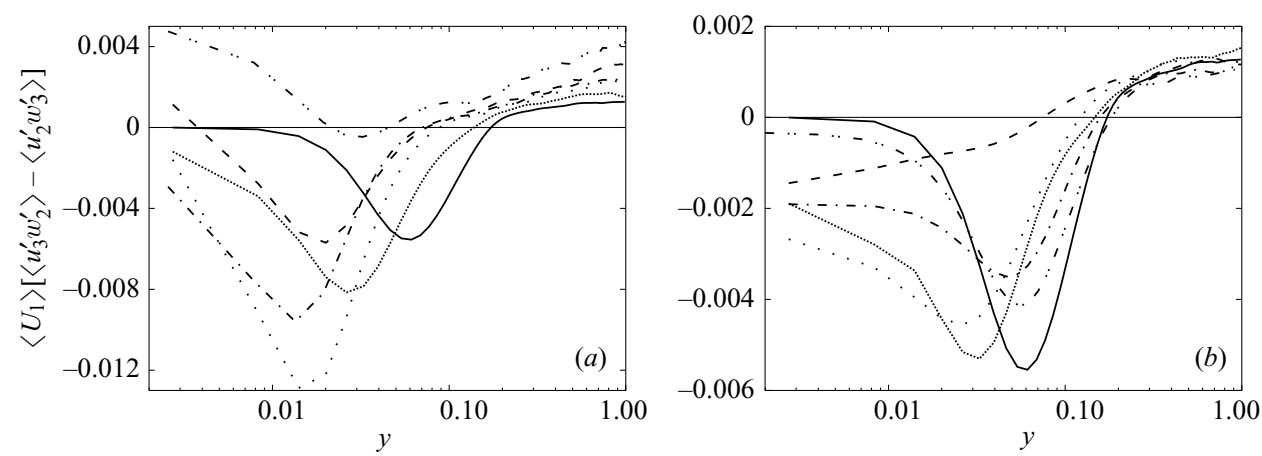

FIGURE 14. Profiles of the rotational energy production (term 1 in (5.1)). (a) Transverse elements: -,$C ; \cdots \cdots \cdots, S_{2 N 1} ; \cdots, R_{2 N 1} ;---, T_{2 N 0} ;---, T_{2 N 1} ;-\cdots-, S_{2 N 7}$. (b) Longitudinal elements:,$- C$; -........, $S_{2 P 1} ; \cdots, R_{2 P 1} ;--, T_{2 P 0} ;---, T_{2 P 1}$; $-\cdots-, T_{1 P 0}$.

The increased organization is also related to the fact that the motion is constrained along the spanwise direction. This is seen clearly in flow visualizations (not presented here).

Near the roughness elements, the profile of term 1 in (5.1) is strongly affected by the roughness geometry and the direction of the energy transfer can change sign. The semi-log plot in figure 14 highlights that, for the geometries $\left(S_{2 N 7}\right)$ which produce a large $\left(\widetilde{u_{2}}\right)_{L}$, the rotational term changes sign in the near-wall region, and thus energy is transferred from small to large scales. This seems reasonable, since, for $S_{2 N 7}$, for example, there are strong ejections outward from the roughness canopy which interact with the incoming large turbulent structures. For the other geometries, on the other hand, the shift towards the wall and the increase of the negative peak imply the increased formation of smaller scales by comparison to the smooth wall. This is the first indication that the elongated structures near a smooth wall tend to disappear. For the longitudinal elements, only for $T_{2 P 1}$ is there a large reduction; this can be appreciated from visualizations of velocity and vorticity components which feature in the rotational production term. This reduction can be better quantified, through the JPDF, to be discussed later. Profiles of the rotational term show that the flow-aligned elements (figure 14b) affect the rotational production less than the transverse elements (figure 14a). Figure 14(a) further shows that for the transverse elements, the positive transfer increases with the disturbance in the outer region. Also, an increase in the magnitude of term 2 in (5.1) is expected because the production must be zero at the crossing point, where $\left\langle u_{1}^{\prime} u_{2}^{\prime}\right\rangle$ and $\partial\left\langle U_{1}\right\rangle / \partial x_{2}$ are zero. Figure $13(a)$ shows that, in the outer region, $\mathscr{P}$ increases with the disturbance owing to a dominance of the rotational production. The final result is that the stresses are larger in the outer region (figure 11a,c,e). For the flow-aligned disturbances (figure 14b), this does not occur and hence the magnitudes of the stresses do not differ from case $C$ (figure $11 b, d, f$ ).

With respect to the near-wall region, it is worth understanding which of the two products, contributing to term 1 in (5.1), is responsible for the change of sign in figure 14(a) for $S_{2 N 7}$ and why there is a large reduction in $T_{2 P 1}$ (figure 14b). Figure $15(a, b)$ shows that, in the near-wall region, $\left\langle u_{3}^{\prime} \omega_{2}^{\prime}\right\rangle$ does not change sign and its magnitude changes in a way that does not simply depend on $\left(\widetilde{u_{2}^{\prime}}\right)_{L}$. Also the correlation $\left\langle u_{3}^{\prime} \omega_{2}^{\prime}\right\rangle$ is similar for $S_{2 N 1}$ and $S_{2 N 7}$. Figure $15(b)$ shows slightly more complex variations for the flow-aligned elements. 

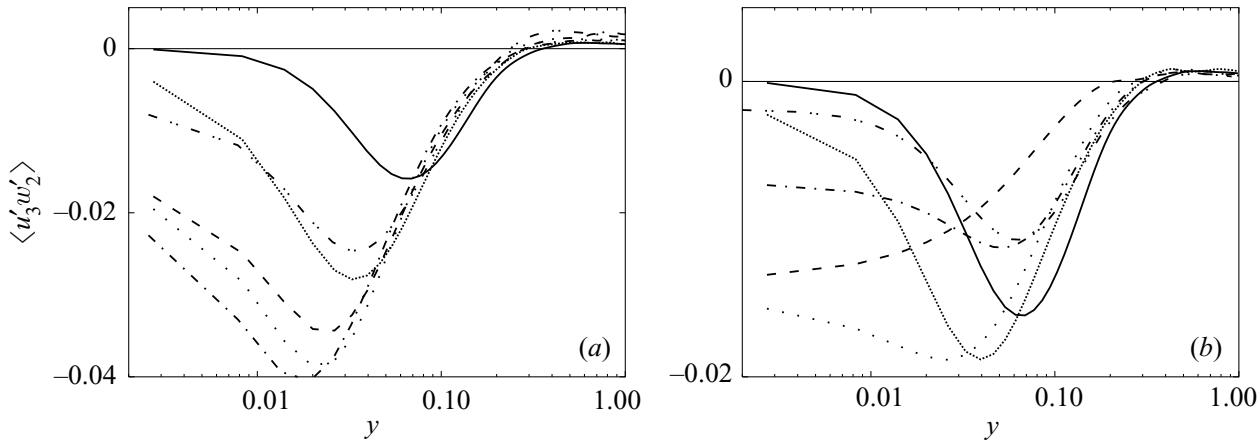

Figure 15. Profiles of $\left\langle u_{3}^{\prime} \omega_{2}^{\prime}\right\rangle$. (a) Transverse elements: -,$C ; \cdots \cdots \cdots \cdots, S_{2 N 1} ; \cdots, R_{2 N 1}$; $--\ldots, T_{2 N 0} ;---, T_{2 N 1} ;-\cdots-, S_{2 N 7}$. (b) Longitudinal elements:,$- C ; \cdots \cdots \cdots, S_{2 P 1}$; $\cdots, R_{2 P 1} ;--, T_{2 P 0} ;---, T_{2 P 1} ;-\cdots-, T_{1 P 0}$.
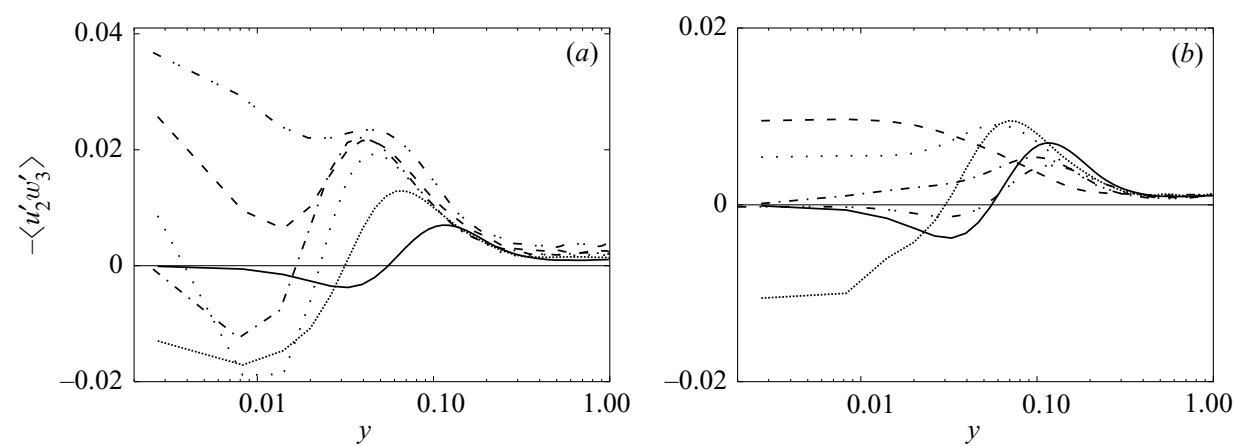

FIGURE 16. Profiles of $-\left\langle u_{2}^{\prime} \omega_{3}^{\prime}\right\rangle .(a)$ Transverse elements:,$- C ; \cdots \cdots \cdots \cdots, S_{2 N 1} ; \cdots, R_{2 N 1}$; ,$--- T_{2 N 0} ;---, T_{2 N 1} ;-\cdots-, S_{2 N 7}$. (b) Longitudinal elements:,$- C ; \cdots \cdots \cdots \cdots, S_{2 P 1}$; ,$R_{2 P 1} ;-\cdots, T_{2 P 0} ;---, T_{2 P 1} ;-\cdots-, T_{1 P 0}$.

The changes in $-\left\langle u_{2}^{\prime} \omega_{3}^{\prime}\right\rangle$ (figure 16) are more complicated. Near the wall, the change of sign is mainly responsible for the change of sign of term 1 . For the flow-aligned elements, the sign of this quantity is different in the near-wall region from that for the smooth-wall channel, reducing the amount of energy lost by $\left\langle u_{3}^{\prime} \omega_{2}^{\prime}\right\rangle$. This effect occurs to a large degree for $T_{2 P 1}$, where the rotational energy production is small and does not show a well-defined peak at a small distance from the wall (figure 15b). The reasons for the changes of these correlations can be better understood through the JPDF. The latter reflects modifications to turbulence structures and is appropriate for discerning changes to velocity-vorticity correlations.

Through the JPDF, it is possible to obtain statistics of any order and, in particular, the correlation coefficients. The JPDF $P\left(\sigma_{1}, \sigma_{2}\right)$, where $\sigma_{i}=\sigma_{i}^{\prime} / \widetilde{\sigma}_{i}$, is such that $\int P\left(\sigma_{i}, \sigma_{j}\right) \mathrm{d} \sigma_{i} \mathrm{~d} \sigma_{j}=1$. The correlation coefficient between $\sigma_{i}$ and $\sigma_{j}$ is given by

$$
\frac{\left\langle\sigma_{i}^{\prime} \sigma_{j}^{\prime}\right\rangle}{\widetilde{\sigma_{i}} \widetilde{\sigma}_{j}}=\iint \sigma_{i}, \sigma_{j} P\left(\sigma_{i}, \sigma_{j}\right) \mathrm{d} \sigma_{i} \mathrm{~d} \sigma_{j} .
$$

The correlation coefficient for the velocity-vorticity tensor, $\Lambda_{i j}$, is defined by

$$
\Lambda_{i j}=\frac{\left\langle u_{i}^{\prime} \omega_{j}^{\prime}\right\rangle}{\widetilde{u}_{i} \widetilde{\omega}_{j}} .
$$



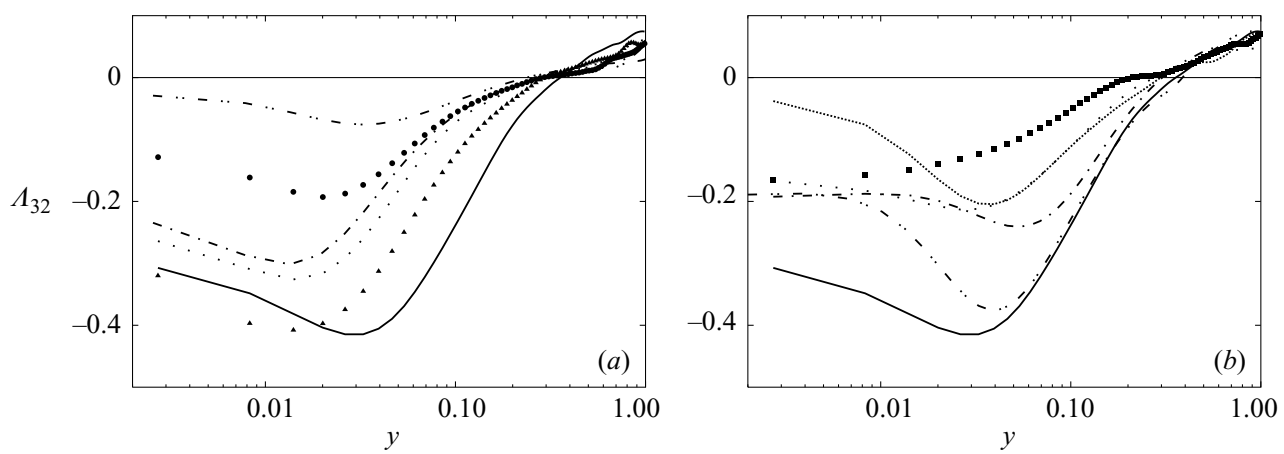

FIGURE 17. Profiles of velocity-vorticity correlation coefficients $\Lambda_{32}$. (a) Transverse elements: ,$- C ; \cdots \cdots \cdots \cdots, S_{2 N 1} ; \bullet, R_{2 N 1} ;---, T_{2 N 0} ; \mathbf{\Delta}, T_{2 N 1} ;-\cdots-, S_{2 N 7} .(b)$ Longitudinal elements: —,$C ; \cdots \cdots, S_{2 P 1} ; \cdots, R_{2 P 1} ;-\cdots, T_{2 P 0} ; \mathbf{\square}, T_{2 P 1} ;-\cdots-, T_{1 P 0}$. Closed symbols indicates the cases investigated through the JPDF.
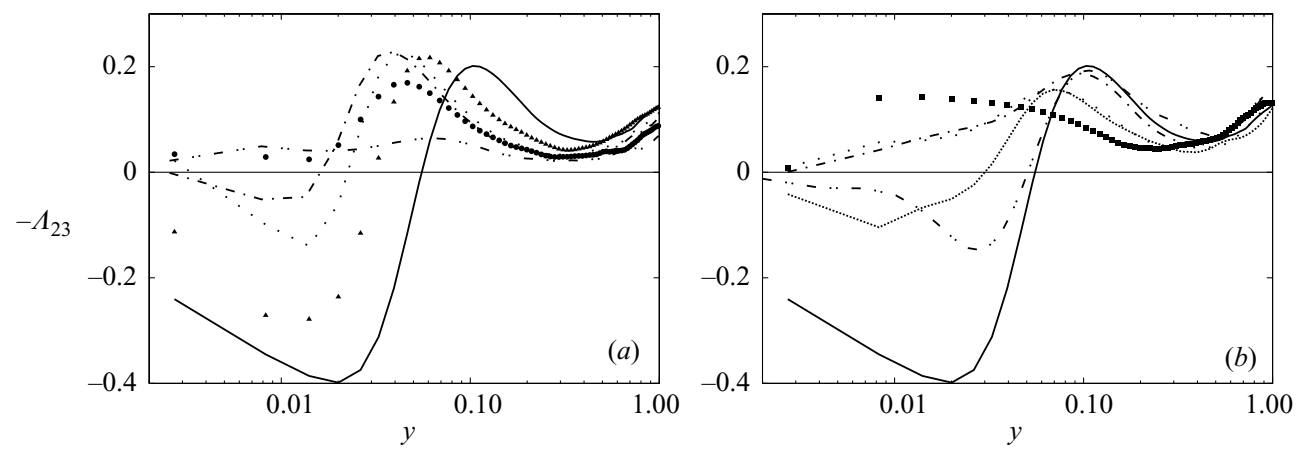

Figure 18. Profiles of correlation coefficients $-\Lambda_{23}$. (a) Transverse elements:,$- C$; $\cdots \cdots, S_{2 N 1} ; \bullet, R_{2 N 1} ;-\cdots, T_{2 N 0} ; \mathbf{\Lambda}, T_{2 N 1} ;-\cdots-, S_{2 N 7}$. (b) Longitudinal elements: $C ; \cdots \cdots \cdots, S_{2 P 1} ; \cdots, R_{2 P 1} ;---, T_{2 P 0} ; \mathbf{\square}, T_{2 P 1} ;-\cdots-, T_{1 P 0}$. Closed symbols indicates the cases investigated through the JPDF.

Profiles of the correlation coefficients differ from those for one-point correlations, but for any particular value of $y$, it is possible to understand via the JPDF which kind of event contributes most to $\left\langle u_{i}^{\prime} \omega_{j}^{\prime}\right\rangle$. The JPDFs corresponding to $S_{2 N 1}, T_{2 N 1}$, $T_{2 P 1}$, (indicated by solid symbols in figures 17-19), are compared with the JPDF for the smooth-wall channel. In addition, it should be noted that $-\Lambda_{23}$ is plotted in figure 18, the magnitude of this quantity having been obtained by summing the contributions from the quadrants. The JPDFs have been evaluated at $y=0.02$. To show that the physics associated with the rotational energy production is complex and is affected significantly by the shape and orientation of the roughness elements, profiles of $\Lambda_{32}$ (figure 17) and $-\Lambda_{23}$ (figure 18) are compared with those of $R_{12}$ in figure 19. The latter correlation coefficients are linked to the formation of low- and high-speed streaks. This comparison shows that, although there are minor differences in the near-wall layer, $R_{12}$ is not too affected by the roughness.

From the JPDF, the integrand in (5.3) can be evaluated, thus allowing the contribution from each quadrant to be quantified. In this section, a prime indicates the ratio of the fluctuation to its r.m.s. value. To understand the production mechanism and its relationship to low- and high-speed streaks, the integrand for $\Lambda_{23}$ 

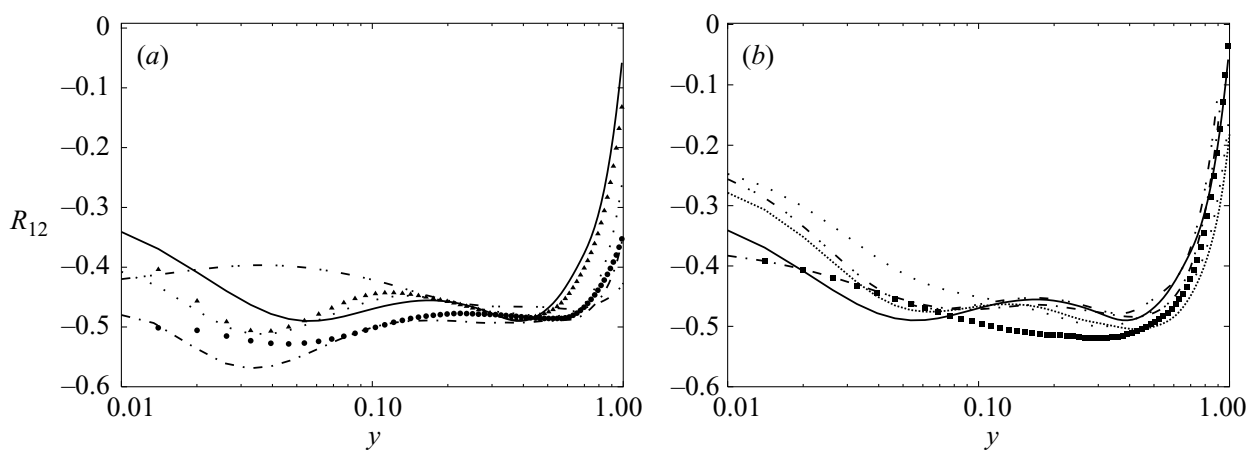

FiguRE 19. Profiles of correlation coefficients $R_{12}$. (a) Transverse elements:

$S_{2 N 1} ; \bullet, R_{2 N 1} ;---, T_{2 N 0} ; \boldsymbol{\Lambda}, T_{2 N 1} ;-\cdots-, S_{2 N 7}$. $(b)$ Longitudinal elements: $-\frac{C}{-} C$; $\cdots \cdots \cdots \cdots, S_{2 P 1} ; \cdots, R_{2 P 1} ;---, T_{2 P 0} ; \mathbf{\square}, T_{2 P 1} ;-\cdots-, T_{1 P 0}$. Closed symbols indicates the cases investigated through the JPDF.

is shown in figure $20(b, e, h, m)$ and for $R_{12}$ in figure $20(c, f, i, n)$. For a smooth wall (figure $20 b$ ) when $\left\langle u_{2}^{\prime} \omega_{3}^{\prime}\right\rangle>0$, the first and third quadrants dominate the other two, and hence contribute to the rotational energy production (figure 16). These quadrants correspond to the ejection $u_{2}^{\prime}>0$ and sweep $u_{2}^{\prime}<0$ events. The ejections produce low-speed streaks $\left(u_{1}^{\prime}<0\right.$ and $\left.\omega_{3}^{\prime}>0\right)$ whereas sweeps produce high-speed streaks. Figure 20(c) shows that the sweeps (fourth quadrant) are the events contributing most to the Reynolds shear stress $\left\langle u_{1}^{\prime} u_{2}^{\prime}\right\rangle$ and hence to the rotational production (third quadrant figure $20 b)$. For the smooth-wall channel, $\Lambda_{23}$ is relatively high $(0.38)$ at $y=0.02$. The contributions from quadrants I to IV are $0.176,-0.052,0.326$ and -0.067 .

For $S_{2 N 1}, P\left(u_{2}^{\prime}, \omega_{3}^{\prime}\right)$ shows that the weak recirculation region increases the number of locations where $u_{2}^{\prime} \approx 0$. The few events with large positive and negative $u_{2}^{\prime}$ correspond to the vertical walls. Figure 20(e) shows that the JPDF becomes more symmetric about $u_{2}^{\prime}=0$ and this implies a decrease in $\Lambda_{23}(0.2365)$. For $S_{2 N 1}$, the contribution from the first quadrant $(0.227)$ is significantly larger than that from the other three $(-0.096,0.180,-0.075)$. Figure $20(e)$ shows that $\omega_{3}^{\prime}$ becomes negatively skewed. The negative value is related to $\partial u_{2} / \partial x_{1}$, produced by the vertical walls of the square bars, rather than by $\partial u_{1} / \partial x_{2}$. For $S_{2 N 1}, R_{12}$ is -0.45 which is close to the smooth-wall channel value $(-0.38)$. Figure $20(f)$ indicates that the second and fourth quadrants are the major contributors to the correlation coefficient. The minor differences between the JPDF for the smooth wall and $S_{2 N 1}$ imply that the low- and high-speed streaks are modified slightly. This is supported by Leonardi et al.'s (2004) two-point velocity correlations.

For geometries generating very strong disturbances $\left(T_{2 N 1}\right)$, figure $20(h)$ shows that $\omega_{3}^{\prime}$ is more negatively skewed, and that the contributions from the second and fourth quadrants increase $(0.189,-0.189,0.100,-0.151)$, leading to a negative $\Lambda_{23}$. From these values, it appears that the strong sweep and ejection events occurring near the trailing and leading edges of the triangular cavity balance each other and the contribution to the low negative rotational energy production term arises from the difference between the fourth and third quadrants. Even for $T_{2 N 1}$, the correlation $R_{12}$ does not change appreciably (figure $20 i$ with contributions $0.0763,-0.246,0.036,-0.373)$. The negative trend for the integrand of $\Lambda_{23}$ is enhanced for $T_{2 P 1}$; in figure $20(\mathrm{~m})$, the contribution of the fourth quadrant increases and that of the first and third decrease; the values are $0.141,-0.189,0.082,-0.175$. 
(a)

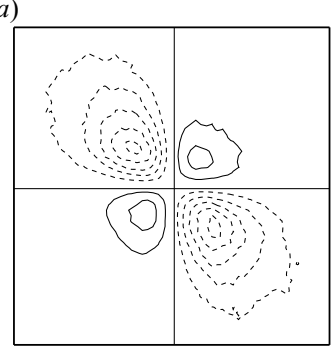

(d)

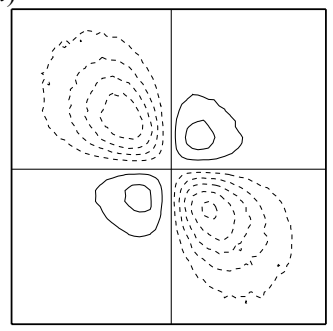

(g)

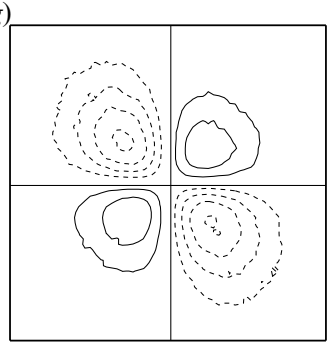

(j)

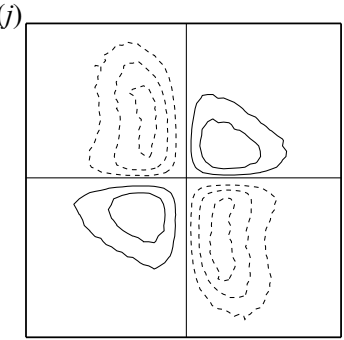

(b)

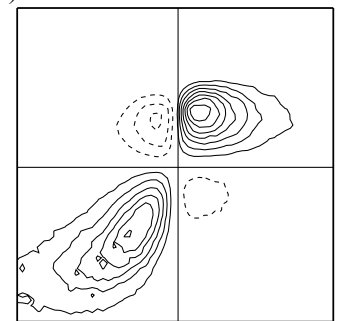

(e)

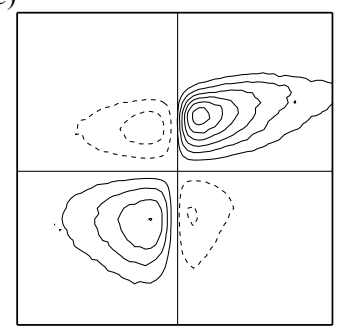

(h)

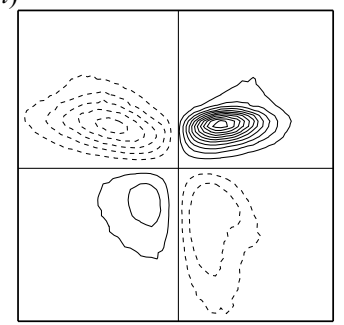

(k)

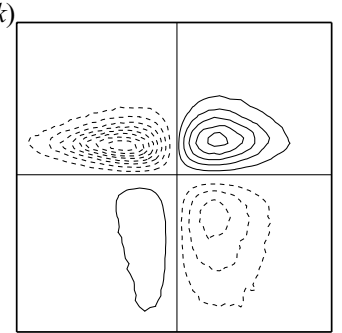

(c)

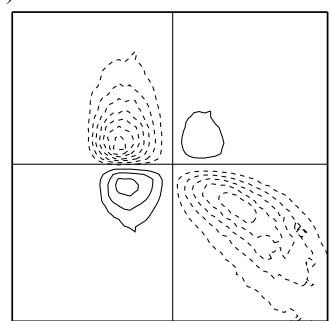

$(f)$

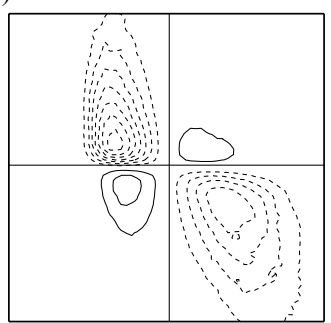

(i)

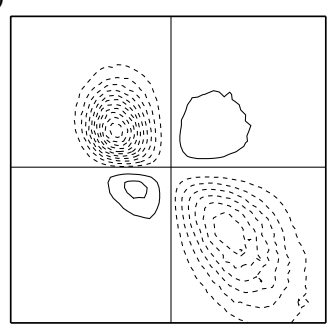

(l)

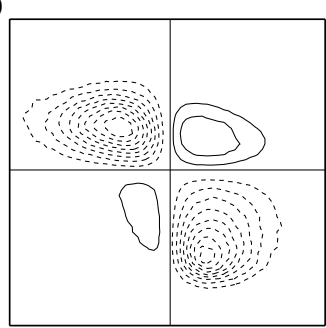

FIGURE 20. Contours of the integrands corresponding to the correlation coefficients: $\Lambda_{32}$ $(a, d, g, l), \Lambda_{23}(b, e, h, m), R_{12}(c, f, i, n)$. Smooth channel $(a, b, c), S_{2 N 1}(d, e, f), T_{2 N 1}(g, h, i)$, $T_{2 P 1}(l, m, n)$. In $a, d, g$ and $l$, the abscissa is $u_{3}^{\prime}$ and the ordinate is $\omega_{2}^{\prime}$. In $b, e, h$ and $m$, the abscissa is $u_{2}^{\prime}$ and the ordinate is $\omega_{3}^{\prime}$. In $c, f, i$ and $n$, the abscissa is $u_{1}^{\prime}$ and the ordinate is $u_{2}^{\prime}$.

The ejections due to the secondary motion inside the triangular riblets, which occur mainly near the sidewalls, contribute less. The sweeps, located mainly in the central region between the apexes of the triangles, contribute to reducing the turbulent kinetic energy production, as discussed in connection with figures 13 and 14 . It follows that the inverse energy cascade from small to large scales is associated with the increased contributions from the second and fourth quadrants. We therefore infer that the inverse energy cascade, caused by the increased intensity of the disturbances (transverse elements), is associated with the second quadrant, i.e. to the events induced by the recirculating region near the reattachment point. 
The other contribution to the $\left\langle u_{3}^{\prime} \omega_{2}^{\prime}\right\rangle U_{1}$ (figure 17) does not change sign near the wall when the shape and orientation of the elements is changed. $\Lambda_{32}$ decreases when the disturbance is increased, in particular for the transverse elements (figure 17a); hence, events contributing to this correlation should be similar. For the cases considered previously, the integrands of (5.3) for $\Lambda_{32}$ are shown in figures $20(a, d, g, l)$. In the absence of disturbances, the second and fourth quadrants contribute most to $\Lambda_{32}$ (figure 20a). When disturbances are present, the contribution from the other two quadrants increases. For the smooth-wall channel, the second and fourth quadrants are related to the streaks. The formation of $\partial u_{1} / \partial x_{3}$ can be explained by a twodimensional model, where $u_{1}$ is convected by $\omega_{1}$ (Orlandi \& Jiménez 1995). A very high $\left(u_{2}^{\prime}\right)$ disturbance is most probably related to the splashing event, described by Leonardi et al. (2004). The leading edge of a roughness element generates a negative $\partial u_{1} / \partial x_{1}$, associated with a positive $\partial u_{3} / \partial x_{3}$. This motion is not organized and promotes the formation of $\partial u_{1} / \partial x_{3}$ of opposite sign to $u_{3}^{\prime}$, the global effect being an improvement in isotropy in the vicinity of the roughness. To understand the difference in $\Lambda_{32}$ between $T_{2 N 1}$ and $T_{2 P 1}$, we report the contributions from the four quadrants: $(0.110,-0.207,0.110,-0.206)$ for $T_{2 N 1}$ and $(0.106,-0.178,0.108,-0.178)$ for $T_{2 P 1}$; i.e. the same value is obtained by a reduction of all the quadrants. From figures $20(\mathrm{~g})$ and $20(\mathrm{l})$, it can be inferred that the PDF of $u_{3}^{\prime}$ does not change and that $\omega_{2}^{\prime}$ is more intermittent for $T_{2 N 1}$ than $T_{2 P 1}$.

The effect on isotropy in the near-wall region, can be further corroborated by analyzing the helicity density, defined by

$$
h^{\prime}=\frac{\boldsymbol{v}^{\prime} \cdot \boldsymbol{\omega}^{\prime}}{\sqrt{\left|\boldsymbol{v}^{\prime}\right|^{2}\left|\boldsymbol{\omega}^{\prime}\right|^{2}}} .
$$

An increase of energy transfer $\boldsymbol{v}^{\prime} \times \boldsymbol{\omega}^{\prime}$ corresponds to a decrease in the helicity density. Since the roughness considered here cannot break the symmetry, the total helicity should remain equal to zero, as found by Rogers \& Moin (1987) for a channel flow and Orlandi (1997) for a pipe flow. To Analyse the alignment between vorticity and velocity in different regions of the channel, the PDF of the helicity density can be used. In previous studies, it was found that, in the near-wall region, the probability that $h^{\prime}$ is zero is large. Near the centre of the channel, where the turbulence is more isotropic (e.g. Kim \& Antonia 1993), there is an equal probability of alignment. The PDF is symmetrical in both regions and the equipartition of the probabilities is characteristic of disordered structures. It should be kept in mind that, in this paper, the interest is in the energy-containing scales. The PDF of the so-called 'worms' studied by Jiménez et al. (1993) in isotropic turbulence should be different. These small structures are outside the scope of this paper.

The above arguments suggest that profiles of total helicity, averaged in the homogeneous directions, do not have any physical interest. Instead, the PDF of $h^{\prime}$ can vary across the channel and may depend on the geometry of the roughness. For the cases discussed previously, the PDFs have been evaluated at two distances from the wall, always in the near-wall region. Figure 21(a) shows that at $y=0.02$ (corresponding to $y^{+}=3.5$ for a smooth-wall channel), the large probability of having orthogonal velocity and vorticity vectors is reduced when the disturbance increases. A distribution close to that for isotropic turbulence is reached when the disturbances are sufficiently strong $\left(R_{2 N 1}, T_{2 N 1}\right.$ and $\left.S_{2 N 7}\right)$. It is worth recalling that, in a smooth-wall channel, $\omega_{3}^{\prime}$ is the largest vorticity component near the wall and $u_{1}^{\prime}$ is larger than the other two components. This explains the poor alignment. Figure 21(b) shows that for the 

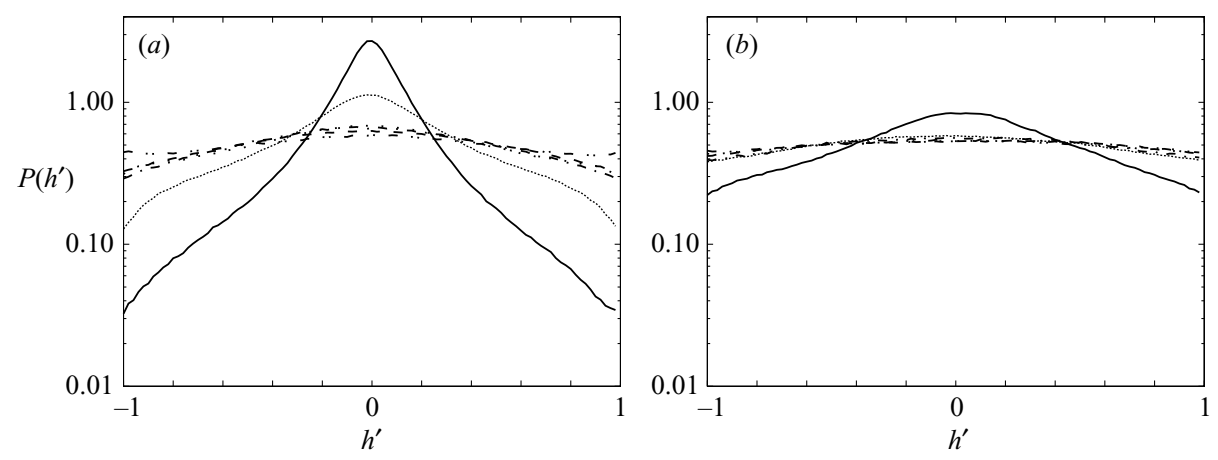

FIGURE 21. Probability density function of the helicity density for transverse elements $(a)$ $y=0.02,(b) y=0.09$. -,$C ; \cdots \cdots \cdots \cdots, S_{2 N 1} ; \cdots, R_{2 N 1} ;---, T_{2 N 0} ;---, T_{2 N 1} ;-\cdots-$ $S_{2 N 7}$.
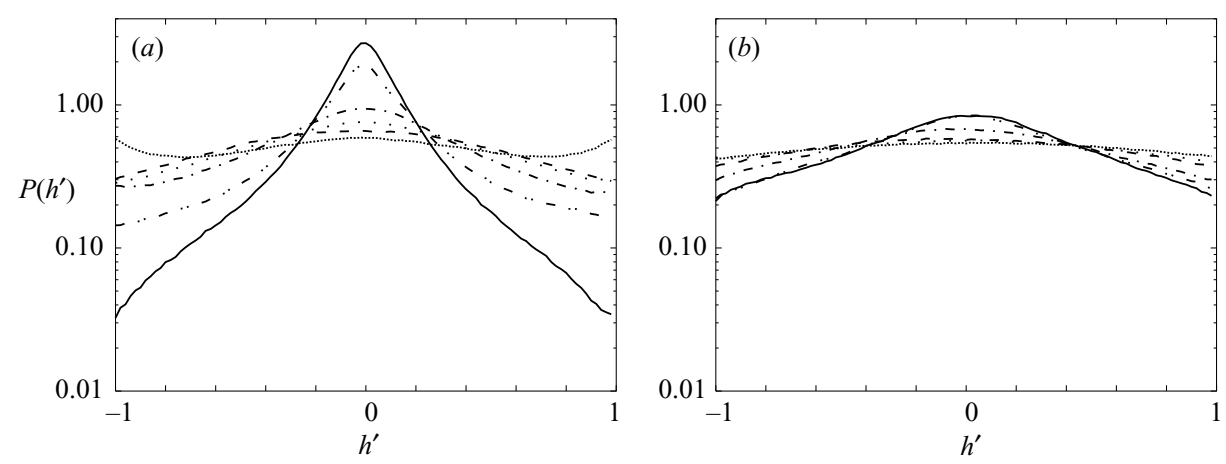

FIGURE 22. Probability density function of the helicity density for longitudinal elements $(a)$ $y=0.02,(b) y=0.09$. -,$C ; \cdots \cdots \cdots \cdots, S_{2 P 1} ; \cdots, R_{2 P 1} ;-\cdot-, T_{2 P 0} ;---, T_{2 P 1} ;-\cdots-$, $T_{1 P 0}$.

smooth-wall channel at $y^{+}=16$, the lack of alignment persists, but is reduced in magnitude and, for all the transverse elements, a state close to isotropy is reached. This is a further check that the roughness produces a near-wall layer where quantities do not vary significantly with distance from the wall. For the flow-aligned elements, the tendency towards isotropy is reduced (figure 22a,b), and for the drag-reducing geometries, the probability of having velocity and vorticity components which are orthogonal increases and persists for greater distances above the roughness crests plane.

\section{Concluding discussion}

A low-Reynolds-number database has been used to understand some aspects of the flow in the vicinity of roughness elements. The study is not exhaustive since three-dimensional roughnesses have not been considered. The mean velocity profiles, normalized by wall variables, were estimated relative to reference quantities in the roughness crests plane. This is possible only in numerical simulations since all quantities can be evaluated in this plane. No satisfactory correlation could be found between $\Delta U^{+}$and the geometrical parameters of the roughness. However, it was observed that the r.m.s. wall-normal velocity is the quantity which best characterizes the rough-wall flows that were considered. This characterization has more general 
validity than any other based solely on geometrical parameters. Although the correlation between $\Delta U^{+}$and $\left(\widetilde{u_{2}}\right)_{L}$ is gratifying, more work is required to demonstrate that this correlation can be extended to industrial and geophysical flows. Orlandi et al. (2003) provided partial evidence for this by showing that the characteristics of roughwall flows were reproduced by simulations of smooth-wall flows after imposing an instantaneous $U_{2}$ distribution, taken from the rough-wall DNS database of Leonardi et al. $(2003 a)$, as a boundary condition. Further evidence may be gathered by imposing a distribution with the same $\left(\widetilde{u_{2}}\right)_{L}$ and random phases across the virtual cavities. If satisfactory results are achieved, it may then be claimed that this constitutes a more general and reliable way of parameterizing the roughness than via $\Delta U^{+}$. The final step could consist in establishing a correspondence between certain kinds of roughness and $\left(\tilde{u_{2}}\right)_{L}$. Previously, the focus has been on determining an appropriate length scale which can account for different types of terrain. The present proposal is more physical, given that almost any code developed for real applications incorporates a turbulence model where $\left(\tilde{u_{2}}\right)_{L}$ can be prescribed as a boundary condition.

Previously, the interest in rough-wall flows has focused mainly on high Reynolds numbers and the possible interrelationship between inner and outer layers. Jiménez (2004) concludes that DNS data cannot yet shed any light on either of these aspects. The issue of how the inner and outer layers interact at high Reynolds numbers remains open, and there appears to be some disagreement among different experiments with regard to how much influence the roughness exerts on the outer region. Leaving aside this issue, we nevertheless believe it is worth investigating how the near-wall region is affected by the shape of the roughness elements. In particular, we have focused on roughness elements with relatively small separations in the streamwise direction, for which the dependence of the roughness function on the roughness geometry is more critical than at large separations.

Even for weak disturbances, we have found that the near-wall behaviour differs from that over a smooth wall. The present DNS data may assist the experimentalist with how to extrapolate measured distributions of $\left\langle u_{1}^{\prime} u_{2}^{\prime}\right\rangle$ and $\left\langle u_{2}^{\prime 2}\right\rangle$ to the roughness crests plane. Such measurements would help to establish whether the parameterization based on $\left(\tilde{u_{2}}\right)_{L}$ could be extended to high Reynolds numbers and to boundary-layer flows. It is certainly of interest that the new parameterization also applies to dragreducing roughness geometries.

To understand why the disturbance penetrates further for transverse than longitudinal elements, vortical structures have been analysed by evaluating the rotational component of the turbulent kinetic energy production, associated with $\boldsymbol{v}^{\prime} \times \boldsymbol{\omega}^{\prime}$. The JPDFs of the correlation coefficients between $\boldsymbol{v}$ and $\boldsymbol{\omega}$ yield useful insight into the inverse energy cascade and the events responsible for this change of sign. Strong support for the tendency towards isotropy near the wall, its connection to drag augmentation and its dependence on the disturbance was provided by the rotational component of the turbulent kinetic energy production and the PDF of the helicity density.

The support of MIUR 60 and $40 \%$ grants is aknoweledged. R. A. A acknowledges the support of the Australian Research Council via both Discovery and IREX grants.

\section{REFERENCES}

Beckert, D. W. \& BARTENWERFER, M. 1989 The viscous flow on surfaces with longitudinal ribs. J. Fluid Mech. 206, 105-129. 
Belcher, S. E., Jerram, N. \& Hunt, J. C. R. 2003 Adjustment of a turbulent boundary layer to a canopy of roughness elements. J. Fluid Mech. 488, 369-398.

Bhaganagar, K., Kim, J. \& Coleman, G. 2004 Effect of roughness on wall-bounded turbulence. Flow Turbulence and Combustion 72, 463-492.

Clauser, F. H. 1954 Turbulent boundary layers in adverse pressure gradients. J. Aero. Sci. 21, 91-109.

Chol, H., Morn, P. \& Kim, J. 1993 Direct numerical simulation of turbulent flow over riblets. J. Fluid Mech. 255, 503-538.

Chu, D. C. \& Karniadakis, G. E. 1993 A direct numerical simulation of laminar and turbulent flow over riblet-mounted surfaces. J. Fluid Mech. 250, 1-42.

Fadlun, E. A., Verzicco, R., Orlandi, P. \& Mohd-Yusof, J. 2000 Combined immersed boundary finite-difference methods for three-dimensional complex flow simulations. J. Comput. Phys. 161, 35-60.

Furuya, Y., Miyata, M. \& Fujita, H. 1976 Turbulent boundary layer and flow resistance on plates roughened by wires. J. Fluids Engng 98, 635-644.

Goldstein, D., Handler, R. \& Sirovich, L. 1995 Direct numerical simulation of turbulent flow over a modelled riblet covered surface. J. Fluid Mech. 302, 333-376.

HinZE, J. O. 1975 Turbulence. McGraw-Hill.

IACCARINO, G. \& VerzICCO, R. 2003 Immersed boundary technique for turbulent flow simulations. Appl. Mech. Rev. 56, 331-347.

JACKSON, P. S. 1981 On the displacement height in the logarithmic profile. J. Fluid Mech. 111, 15-25.

JimÉnez, J. 2004 Turbulent flows over rough walls. Annu. Rev. Fluid Mech. 36, 173-196.

Jiménez, J., Wray, A. A., Saffman, P. G. \& Rogallo, R. S. 1993 The structure of intense vorticity in isotropic turbulence. J. Fluid Mech. 255, 65-90.

Kim, J. \& Antonia, R. A. 1993 Isotropy of the small scales of turbulence at low Reynolds number. J. Fluid Mech. 251, 219-238.

Kim, J., Moin, P. \& Moser, R. 1987 Turbulence statistics in fully developed channel flow at low Reynolds number. J. Fluid Mech. 177, 133-166.

Krogstad, P.-Å. \& Antonia, R. A. 1999 Surface roughness effects in turbulent boundary layers. Exps. Fluids 27, 450-460.

Leonardi, S., Orlandi, P., Smalley, R. J., Djenidi, L. \& Antonia, R. A. $2003 a$ Direct numerical simulations of turbulent channel flow with transverse square bars on the wall. J. Fluid Mech. 491, 229-238.

Leonardi, S., Mostarda, E., Orlandi, P. \& Antonia, R. A. $2003 b$ Budgets of Reynolds Stress in a Rough Wall Channel Direct and Large-Eddy Simulation V. (ed. B. J. Geurts, R. Friedrich \& O. Metais), pp. 509-516. Kluwer.

Leonardi, S., Orlandi, R. J., Djenidi, L. \& Antonia, R. A. 2004 Structure of turbulent channel flow with square bars on one wall. Intl J. Heat Fluid Flow 25, 384-392.

Leonardi, S., Orlandi, R. J. \& Antonia, R. A. 2005 A method for determining the frictional velocity in a turbulent channel flow with roughness on the bottom wall. Exps. Fluids 33, $31-37$.

Moore, W. L. 1951 An experimental investigation of boundary layer development along a rough surface. PhD thesis, State University of Iowa.

NikuRadSe, J. 1933 Stromungsgesetze in rauhen Rohren. Forsch. Arb. Ing.-Wes. 361.

ORLANDI, P. 1989 Numerical solution of 3-D flows periodic in one direction and with complex geometries in 2-D. Annual Research Briefs, Center for Turbulence Research 1989, pp. 215-230.

OrLand, P. 1997 Helicity fluctuations in rotating and non-rotating pipes. Phys. Fluids A 9, 2045 2056.

Orlandi, P. 2000 Fluid Flow Phenomena: A Numerical Toolkit. Kluwer.

Orlandi, P. \& JimÉnez, J. 1995 On the generation of turbulent wall friction. Phys. Fluids 6, $643-3600$.

ORLANDI, P. \& LEONARDI, S. 2001 DNS: a tool for numerical experiments. Turbulence structures and modulation (ed. A. Soldati \& R. Monti), pp. 93-118. Springer.

Orlandi, P., Leonardi, S., Tuzi, R. \& Antonia, A. R. 2003 DNS of turbulent channel flow with wall velocity disturbances. Phys. Fluids 15, 3497-3600.

Perot, B. \& Moin, P. 1995 Shear-free turbulent boundary layers. Part 1. Physical insights into near-wall turbulence. J. Fluid Mech. 295, 199-227. 
Perry, A. E., Schofield, W. H. \& Joubert, P. N. 1969 Rough wall turbulent boundary layers. J. Fluid Mech. 37, 383-413.

Rogers, M. M. \& Moin, P. 1987 The structure of the vorticity field in homogeneous turbulent flows. J. Fluid Mech. 176, 33-66.

Uzkan, T. \& Reynolds, W. C. 1967 A shear-free turbulent boundary layer. J. Fluid Mech. 28, 803-821.

Vukoslavcevic, P. Wallace, J. M. \& Balint, J. L. 1992 Viscous drag reduction using streamwise aligned riblets. AIAA J. 30, 1119-1122.

Walsh, M. J. 1990 Riblets. In Viscous Drag Reduction in Boundary Layers (ed. D. Bushnell \& J. Hefner). Progress in Astronautics and Aeronautics, vol. 123, pp. 203-259. AIAA.

Ye, T., Mittal, R., Udaykumar, H. S. \& Shy, W. 1999 An accurate Cartesian grid method for viscous incompressible flows with complex immersed boundaries. J. Comput. Phys. 156, 209-240. 